\title{
Statistics of severe tornadoes and severe tornado outbreaks
}

\author{
B. D. Malamud ${ }^{1}$ and D. L. Turcotte ${ }^{2}$ \\ ${ }^{1}$ Department of Geography, King's College London, Strand, London, WC2R 2LS, UK \\ ${ }^{2}$ Department of Geology, University of California, Davis, CA 95616, USA \\ Correspondence to: B. D. Malamud (bruce.malamud@kcl.ac.uk)
}

Received: 30 December 2011 - Published in Atmos. Chem. Phys. Discuss.: 7 March 2012

Revised: 9 August 2012 - Accepted: 15 August 2012 - Published: 20 September 2012

\begin{abstract}
The standard measures of the intensity of a tornado in the USA and many other countries are the Fujita and Enhanced Fujita scales. These scales are based on the damage that a tornado causes. Another measure of the strength of a tornado is its path length of touchdown, $L$. In this study we consider severe tornadoes, which we define as $L \geq 10 \mathrm{~km}$, in the continental USA (USA Storm Prediction Center Severe Weather Database). We find that for the period 1982-2011, for individual severe tornadoes $(L \geq 10 \mathrm{~km})$ : (i) There is a strong linear scaling between the number of severe tornadoes in a year and their total path length in that year. (ii) The cumulative frequency path length data suggests that, not taking into account any changing trends over time, we would expect in a given year (on average) one severe tornado with a path length $L \geq 115 \mathrm{~km}$ and in a decade (on average) one severe tornado with a path length $L \geq 215 \mathrm{~km}$. (iii) The noncumulative frequency-length statistics of severe tornado touchdown path lengths, $20<L<200 \mathrm{~km}$, is well approximated by an inverse power-law relationship with exponent near 3 . We then take the total path length of severe tornadoes in a convective day (12:00-12:00 UTC), $L_{\mathrm{D}}$, as a measure of the strength of a 24-h USA tornado outbreak. We find that: (i) For 1982-2011, the number of severe tornadoes in a USA convective day outbreak has a strong power-law relationship (exponent 0.80 ) on the convective day total path length, $L_{\mathrm{D}}$. (ii) For 1952-2011, the cumulative frequency path length data for severe tornado outbreaks suggests that we would expect in a given year (on average) one daily severe tornado outbreak with total path length $L_{\mathrm{D}} \geq 480 \mathrm{~km}$ and in a decade (on average) one daily severe tornado outbreak with a total path length $L_{\mathrm{D}} \geq 1200 \mathrm{~km}$. (iii) For 1982-2011, the noncumulative frequency-length statistics of tornado outbreaks, $10<L_{\mathrm{D}}<1000 \mathrm{~km} \mathrm{~d}^{-1}$, is well approximated by an inverse power-law relationship with exponent near 1.8. Finally, we
\end{abstract}

consider the frequency path-length scaling of severe tornadoes $(L \geq 10 \mathrm{~km})$ during two tornado outbreaks, 27 April 2011 (67 severe tornadoes) and 25 May 2011 (16 severe tornadoes), and find similar statistical distributions with robust scaling. We believe that our robust scaling results provide evidence that touchdown path lengths can be used as quantitative measures of the systematic properties of severe tornadoes and severe tornado outbreaks.

\section{Introduction}

This paper introduces and tests hypotheses for quantifying the intensities of severe tornadoes and severe tornado outbreaks. Our approach is in analogy to the historic evolution of the qualitative (damage-based) Mercalli scale relative to the quantitative (displacement-based) Richter scale for earthquakes. The Fujita and Enhanced Fujita scales, currently used for tornadoes, are based qualitatively on damage, from which wind intensity and other quantitative measures are estimated. Ideally, tornado intensities would be based on the distribution of velocities in a tornado. However, as noted by Doswell et al. (2009), systematic and high-resolution Doppler remote sensing of wind velocities in tornadoes is not possible at this time.

In this paper, we will use the tornado path length $L$ as a quantitative measure of tornado intensity and will use as our definition of a severe tornado, those with $L \geq 10 \mathrm{~km}$. A detailed study of the statistical relationship between tornado path lengths $L$ and Fujita scale intensities has been given by Brooks (2004). In this paper we extend his approach, to further develop individual tornado and tornado outbreak path length statistics to aid in improving our understanding of tornado climatology. 
The standard measure of the tornado intensity is the Fujita scale (Fujita, 1971, 1981; Fujita and Pearson, 1973) also referred to as the Fujita-Pearson scale. This scale was introduced in the 1970s as a measure of tornado intensity, with tornadoes rated on a scale of F0 to F5 based on the maximum damage caused along a given tornado path. In the United States, the enhanced Fujita scale replaced the Fujita scale for intensity assessment on 1 February 2007, using different and more specific criteria for assessment (Potter 2007). In terms of applications, the Fujita scale and the enhanced Fujita scale are considered equivalent, so that in the remainder of this paper we will refer to the Fujita scale.

It is of interest to compare probabilistic risk assessment for tornadoes with that of earthquakes. From 1880 until 1935 the Mercalli scale was used to determine the intensity of earthquakes. The Mercalli scale was based on damage, and is in direct analogy to the Fujita scale for tornadoes (Doswell et al., 2009). In 1935 the Mercalli scale was replaced by the Richter scale (Richter, 1935) as the accepted measure of earthquake intensity. The Richter scale utilized the displacement amplitudes obtained from regional seismographs to quantify the ground shaking responsible for damage and deaths. In 1979 seismograph displacements were used to directly determine the moment (radiated energy) of an earthquake (Hanks and Kanamori, 1979). Earthquake moments are then converted to moment magnitudes because of the public acceptance of the Richter magnitude scale. The association between earthquake and tornado risk assessments has also been discussed by Schielicke and Névir (2011).

The principle purpose of this paper is to carry out a study of the statistics of tornado touchdown path lengths, $L$. In Sect. 2 we discuss the data, present and discuss frequencypath length statistics for all path lengths $L$, and then relationships between path lengths $L$ and Fujita scale intensities, F0 to F5. Because of data quality, we will consider only severe tornadoes, and utilize two definitions: (i) Tornadoes having touchdown path lengths $L \geq 10 \mathrm{~km}$ (and all Fujita scale intensities F0 and greater); (ii) Only strong (F2 and F3) and violent (F4 and F5) tornadoes (and all $L \geq 0 \mathrm{~km}$ ). These two definitions have approximately the same number of tornadoes for the period considered. However, only about one half of the severe tornadoes are included in both definitions. In Sect. 3, we begin by considering the statistics of individual severe tornadoes $(L \geq 10 \mathrm{~km})$ during the period 1982 2011, including the statistics of severe tornado occurrence as a function of the hour of day, and day of the year. We will then consider, using both definitions of severe tornadoes, the total number vs. path length per year, and will conclude that the path-length definition for severe tornadoes $(L \geq 10 \mathrm{~km})$ is preferable, which we will use for the rest of our studies. We will close Sect. 3 by showing that the probability of a given length $L$ occurring for severe tornadoes $(L \geq 10 \mathrm{~km})$ scales with $L$.

Then in Sect. 4, we extend our studies of individual severe tornadoes to the total path length of severe tornadoes in a convective day (12:00-12:00 UTC (Coordinated Universal Time)), $L_{\mathrm{D}}$, which we take as a measure of the strength of a continental USA tornado outbreak in a one-day period. Doswell et al. (2006) have suggested that $L_{\mathrm{D}}$ is the preferred measure of the strength of a tornado outbreak. Verbout et al. (2006) also discuss the number of tornadoes above a given threshold in a convective day as a measure of the strength of a tornado outbreak. We show that the number of tornadoes in a convective day scales with the total length of tornadoes in that convective day, and consider the probability of a given outbreak total path length $L_{\mathrm{D}}$ occurring. We also consider the cumulative frequency-path length statistics of severe tornadoes during two convective day tornado outbreaks. Finally, in Sect. 5, we discuss our approach.

\section{Data}

In this paper we consider the statistics of tornado occurrence in the continental United States. We use six decades of the National Weather Service (NWS) Storm Prediction Centre (SPC) database of tornadoes (McCarthy, 2003) for the time period 1952 to 2011 (NOAA, 2012). For the 56749 tornado records during this period, information includes (in most cases) tornado date, time, location (latitude, longitude, county, state), Fujita scale (or enhanced Fujita scale) intensity, injuries, fatalities, damage, and touchdown path length and width. A number of records were removed based on the listed values of tornado path length, $L$. In the original database, tornadoes that touched down in more than one state had a path length record for each state, and another one for the entire summed path length for the multiple states. Therefore 990 values ( $1.7 \%$ of the original dataset records) were removed that were one part of a multi-state record (the multistate record was left in place). Also removed from the original dataset were 56 values $(0.1 \%$ of the original dataset records) with path lengths that were $L=400,300,200,100$, $80,50,30,25,20,15,10,8$ miles (the original units of the database), but where the starting and ending latitude and longitude coordinates were listed as being exactly the same (i.e., 0 miles traversed). It was assumed that these records were in error due to being exactly on multiples of 100 (or 10) and having zero path length based on touchdown starting/ending coordinates. The final database used here for 1952-2011 (all touchdown path lengths $L$ ), had a total of 55703 tornadoes.

We first consider the frequency-path length statistics for all tornadoes. In Fig. 1 we give the cumulative number of tornadoes per year $N_{\mathrm{c}}$ with touchdown path lengths greater than $L$, as a function of $L$. Values are given for six $10-\mathrm{yr}$ periods, 1952-2011. In Fig. 1a we consider all tornadoes of any path length $L$ (55703 values) and in Fig. 1b just those tornadoes with $L \geq 10 \mathrm{~km}$ (8018 values). There is a clear visual difference between the three 10 -yr frequencysize distributions for 1952-1981 compared to the three 10yr frequency-size distributions during the period 1982-2011. 

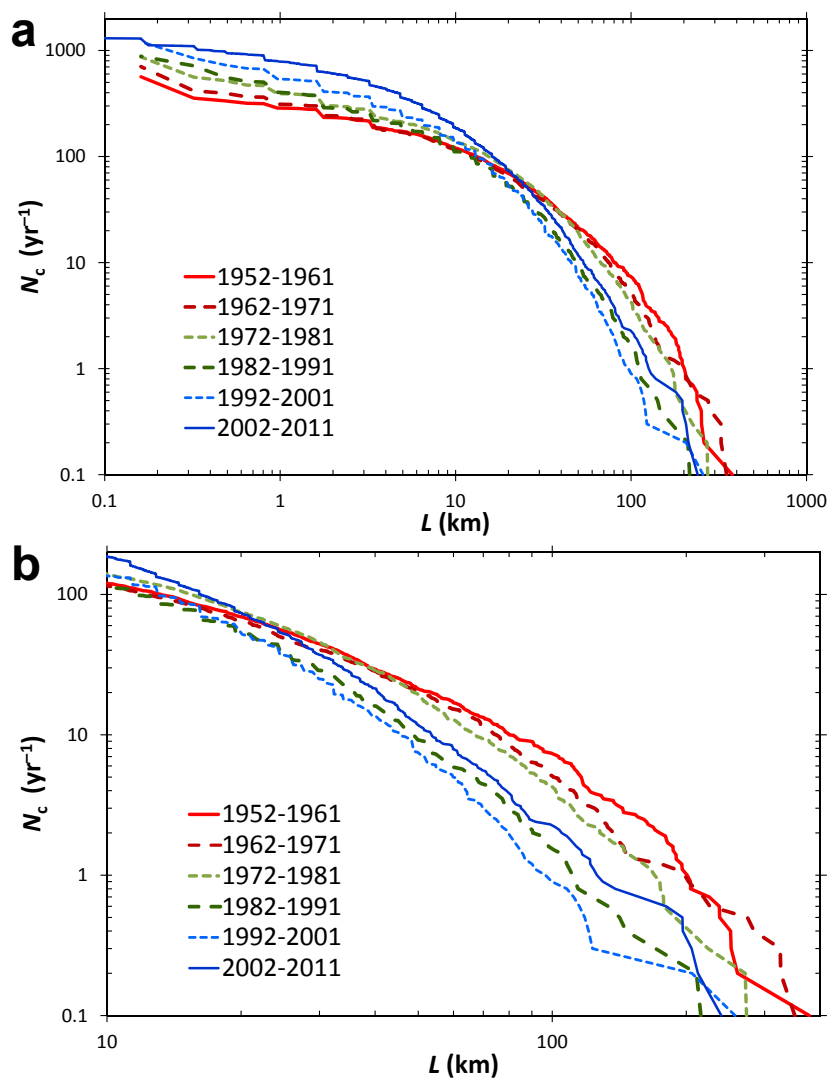

Fig. 1. Cumulative number of continental USA tornadoes per year $N_{\mathrm{c}}$ with path lengths greater than or equal to $L$, given as a function of $L$. Data are given for six 10-yr periods from 1952-2011. (a) All tornadoes. (b) Data for $L \geq 10 \mathrm{~km}$, which is one definition we use in this paper as a severe tornado. Tornado path length data $L$ are from NOAA (2012).

Many fewer long path lengths were recorded in the later 30yr period and there has been, decade by decade, a more systematic reporting of weak tornadoes over time. Schaefer et al. (2002), Brooks (2004) and Verbout et al. (2006) have previously noted these differences and suggested that one of the reasons for the difference in completeness is related to the beginning of real-time touchdown surveys. Beginning in the early 1980s, a Warning Preparedness Meteorologist (WPM) was assigned to 52 Weather Service Forecast Offices; the WPM was responsible for tornado surveys in a specified region (McCarthy, 2003). This contrasts with the earlier period (1952-1981), during which tornado touchdown path lengths were primarily determined from newspaper accounts, which appear to have systematically over-stated the actual values (McCarthy, 2003). One explanation he gave for this effect was that several tornadoes with shorter path lengths were often combined to give a single long path length. Field surveys have certainly given more accurate data on tornado path lengths. Other factors for a more systematic reporting of weak tornadoes over time (Brooks and Doswell, 2001)

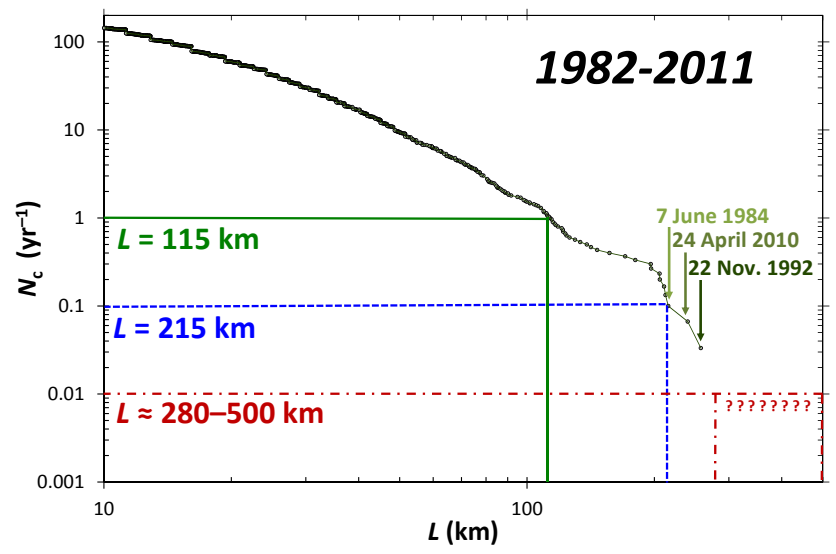

Fig. 2. Cumulative number of continental USA tornadoes per year $N_{\mathrm{c}}$ with path lengths greater than or equal to $L$ is given as a function of $L$. The data (NOAA, 2012) are for the period 1982-2011 and for $L \geq 10 \mathrm{~km}$, defined in this paper to be severe tornadoes. The three longest path lengths are identified by vertical arrows. Using this data, rough estimates are made for the expected 1-yr, 10-yr, and 100 -yr tornadoes.

include a general improvement of public awareness and increasing urbanization.

Based on the data shown in Fig. 1, we will consider, for most of our studies in the remainder of this paper, just those tornadoes that occurred during the period 1982-2011 (34328 values, all touchdown path lengths $L$ ). It can be seen from Fig. 1 that the decadal frequency-length statistics for the three periods 1982-1991, 1992-2001, and 20022011 are reasonably self-consistent. In Fig. 2 we combine the last three decades (1982-2011), and only for tornadoes with touchdown path lengths $L \geq 10 \mathrm{~km}$, we give the cumulative number of tornadoes per year $N_{\mathrm{c}}$ with path lengths greater than $L$, as a function of $L$. During this 30 -yr period, the longest path length $L=257 \mathrm{~km}$ occurred on 22 November 1992, the 2nd longest $L=240 \mathrm{~km}$ on 24 April 2010, and the 3rd longest $L=216 \mathrm{~km}$ on 7 June 1984. It is interesting to note that the longest path length during the very active 2011 USA tornado season ranked 4th with $L=212 \mathrm{~km}$.

As illustrated in Fig. 2 the cumulative frequency-length data can be used to give a rough estimate of the hazard of severe tornado occurrence. Based on our data, we estimate the annual tornado touchdown path length to be $L \geq 115 \mathrm{~km}$. In other words, on average over many years, not taking into account any changing trends over time, we would expect in any given year one tornado with a path length $L \geq 115 \mathrm{~km}$. The ten year tornado (the longest path length or greater expected in a 10 -yr period) is $L \geq 215 \mathrm{~km}$. An extrapolation of the curve is difficult for longer recurrence periods, as the shape of the statistical distribution for the largest lengths is not well defined. Our visual estimate based on the data given in Fig. 2 for the 100-yr tornado (the longest path length or greater expected in a $100-\mathrm{yr}$ period) is in the range $280-500 \mathrm{~km}$. This 


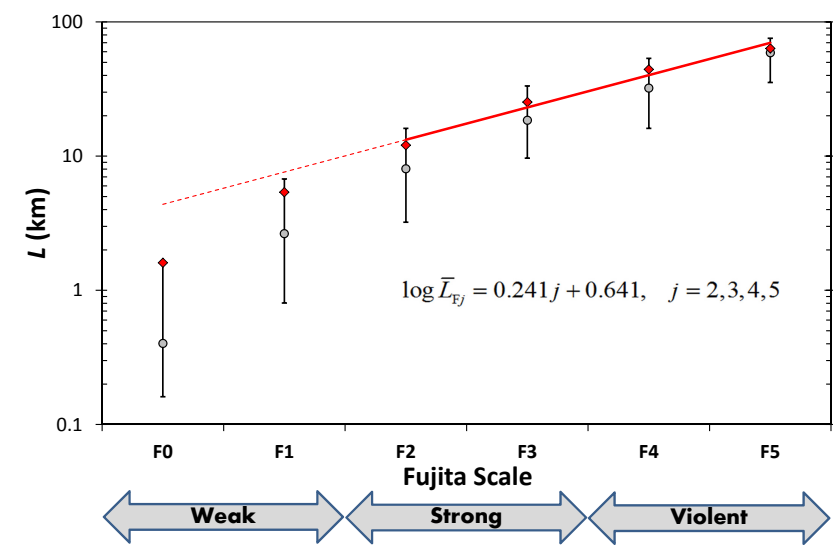

Fig. 3. Continental USA tornado touchdown path length statistics as a function of Fujita (or Enhanced Fujita) scale intensity F0, F1, ..., F5, for the time period 1982-2011, with all path lengths $L$ considered. Included are the mean path lengths $\overline{L_{\mathrm{F} j}}$ (red diamonds) for each Fujita scale intensity $(\mathrm{F} j, j=0,1,2, \ldots, 5)$, median values (grey circles), and the 75th and 25th percentile (upper and lower horizontal lines). Also given (thick red line) is the best-fit to the mean values for strong (F2, F3) and violent (F4, F5) tornadoes (Eq. 1). Tornado path length and Fujita scale intensity data are from NOAA (2012).

extrapolation of the data is uncertain for these extreme values.

The emphasis in this paper is on the statistics of tornado path lengths as a measure of tornado intensity. Since the standard measure of tornado intensity is the Fujita scale, it is important to consider relations between the Fujita scale intensity and tornado path lengths. Brooks (2004) has studied in detail the statistical distribution of path lengths for F0 to F5 tornadoes. He carried out his study for all tornadoes from 1950-2001. In Fig. 3, we relate the statistical measures of tornado touchdown path lengths $L$ as a function of Fujita scale for intensities F0 to F5, for all tornadoes 1982-2011. For each Fujita scale intensity, we give the mean touchdown path length (red diamonds), median (grey circles), and the 75 th and 25 th percentiles (upper and lower horizontal lines). For F2 to F5 (i.e., strong to violent) tornadoes, the best-fit linear trend line (thick red line) to the mean path length values is:

$\log \overline{L_{\mathrm{F} j}}=0.241( \pm 0.026) j+0.641, j=2,3,4,5$,

where $\overline{L_{\mathrm{F} j}}$ is the mean of all tornado path lengths $L$ at a given Fujita scale intensity, $\mathrm{F} j, j=2,3,4,5$, and the uncertainties are \pm 1 s.e. (standard error) on the slope. Eq. (1) can be written as:

$\frac{\overline{L_{\mathrm{F} j}+1}}{\overline{L_{\mathrm{F} j}}}=10^{0.241( \pm 0.026)}=1.64-1.85, j=2,3,4,5$.

That is, the mean path length of an F3 tornado is 1.64-1.85 times longer than the mean path length of an F2 tornado, and the mean path length of an F5 tornado is $1.64^{3}-1.85^{3}=4.4-$ 6.3 times longer than the mean path length of an F2 tornado. In Table 1 we compare our mean path lengths for the period 1982-2011 with those given by Brooks (2004) for the period 1950-2001, and find good agreement, despite the different time periods considered and the differences in data completeness. We also include the early work of Fujita and Pearson (1973) where they gave a range of touchdown path lengths associated with specific Fujita scale intensities. Their intensity values were based on a small sample of tornadoes and we believe over-estimate the path length values for each $\mathrm{Fu}$ jita scale.

From Fig. 3 we see that reasonably good scaling of the mean touchdown path lengths (red diamonds) as a function of Fujita scale intensity is obtained for tornadoes F2 to F5. The deviation from this scaling for F0 and F1 tornadoes is likely due to limitations of the Fujita scale for weak tornadoes, measurement problems with determining path lengths for these weak tornadoes and incomplete reporting of smaller tornado path lengths. For these reasons, one possible definition for severe tornadoes, in terms of the Fujita scale, includes those intensities that are F2 or larger (i.e., "strong" and "violent" tornadoes). Since our studies are based on tornado path lengths, an alternative definition for a severe tornado, which we will use later, is a tornado that has a touchdown path length $L \geq 10 \mathrm{~km}$. We will discuss these two severe tornado definitions in Sect. 3. In terms of path lengths, we see from Fig. 3 and Table 1 that on average, the minimum touchdown path length value in our definition of severe tornadoes ( $L \geq 10 \mathrm{~km}$ ) approximately coincides with F2 (strong) tornadoes at $L=12.1 \mathrm{~km}$, with weak (F0, F1) tornadoes having path lengths that significantly deviate from the scaling seen for strong (F2, F3) and violent (F4, F5) tornadoes.

We note that if one considers the path length statistics vs. Fujita scale intensities for only the final 5-yr period of the record (2007-2011), and performs a similar analysis as in Fig. 3, the resultant best-fit linear trend line is $\log \overline{L_{\mathrm{F} j}}=$ $0.251( \pm 0.027) j+0.684, j=2,3,4,5$, which is very similar to the values in Eq. (1) (1982-2011). However, in contrast to Fig. 3 (1982-2011) where the mean path lengths for $\mathrm{F} 1$ and F0 (red diamonds), $\overline{L_{\mathrm{F} 1}}=5.4 \mathrm{~km}$ and $\overline{L_{\mathrm{F} 0}}=1.6 \mathrm{~km}$, deviated strongly from the best-fit linear trend extrapolation, the mean path length values for $2007-2011$ are $\overline{L_{\mathrm{F} 1}}=7.2 \mathrm{~km}$ and $\overline{L_{\mathrm{F} 0}}=2.2 \mathrm{~km}$, which are closer to their best-fit linear trend extrapolation. We would suggest then that a significant part of the deviation of F0 and F1 tornadoes from the scaling relationship between mean path length vs. Fujita scale intensity (for F2, F3, F4, F5), is the underreporting of weak tornadoes in earlier years of the record. We would further suggest that as the recording of weak tornadoes improves even more, that this scaling relationship of mean path length and Fujita scale intensity will become more evident for not just strong and violent tornadoes, but also for weak tornadoes (i.e., the red diamonds in Fig. 3 will approach the dashed extrapolation of the scaling). However, for the purposes of the analyses 
Table 1. Continental USA tornado touchdown path lengths $L$ as a function of Fujita (or Enhanced Fujita) scale intensities $\mathrm{F} j, j=0,1$, $2, \ldots, 5$. In the 2nd column, the range of path lengths $L(\mathrm{~km})$ given by Fujita and Pearson (1973) are given. In the 3rd and 4th columns, the mean tornado path lengths $\overline{L_{\mathrm{Fj}}}$ in the continental USA given by Brooks (2004) and in this paper (Fig. 3) are given, with all path lengths $L$ considered.

\begin{tabular}{llll}
\hline $\begin{array}{l}\text { Fujita Scale } \\
\text { Intensity }\end{array}$ & $\begin{array}{l}\text { Fujita and Pearson (1973) } \\
\text { Range of tornado path } \\
\text { lengths } L(\mathrm{~km})\end{array}$ & $\begin{array}{l}\text { Brooks }(2004) \\
1950-2001 \\
\text { Mean tornado path } \\
\text { length } \overline{L_{\mathrm{F} j}}(\mathrm{~km})\end{array}$ & $\begin{array}{l}\text { This paper } \\
\text { 1982-2011 } \\
\text { Mean tornado path } \\
\text { length } \overline{L_{\mathrm{F} j}}(\mathrm{~km})\end{array}$ \\
\hline F0 & $0.5-1.5$ & 1.4 & 1.6 \\
F1 & $1.6-5.0$ & 4.7 & 5.4 \\
F2 & $5.1-15.9$ & 10.7 & 12.1 \\
F3 & $16.0-50$ & 22.5 & 25.3 \\
F4 & $51-159$ & 43.6 & 44.3 \\
F5 & $160-500$ & 54.6 & 64.4 \\
\hline
\end{tabular}

Table 2. Number and percentage of continental USA tornado path lengths from 1982-2011, $L<10 \mathrm{~km}$ and $L \geq 10 \mathrm{~km}$ (i.e., "severe" tornadoes as defined in this paper), as a function of Fujita scale intensities F. Data are from NOAA (2012).

\begin{tabular}{|c|c|c|c|}
\hline $\begin{array}{l}\text { Fujita Scale } \\
\text { Intensity }\end{array}$ & $\begin{array}{l}\text { Tornadoes with } \\
L<10 \mathrm{~km} \\
\text { \# (\% in Fujita category) }\end{array}$ & $\begin{array}{l}\text { "Severe" tornadoes } \\
\text { with } L \geq 10 \mathrm{~km} \\
\text { \# (\% in Fujita category) }\end{array}$ & $\begin{array}{l}\text { All tornadoes } \\
(L \geq 0 \mathrm{~km}) \\
\#(\% \text { in Fujita category })\end{array}$ \\
\hline F0 & $19255(97 \%)$ & $521(3 \%)$ & $19776(100 \%)$ \\
\hline F1 & $8552(84 \%)$ & $1616(16 \%)$ & $10168(100 \%)$ \\
\hline $\mathrm{F} 2$ & $1902(59 \%)$ & $1305(41 \%)$ & $3207(100 \%)$ \\
\hline F3 & $273(29 \%)$ & $674(71 \%)$ & $947(100 \%)$ \\
\hline $\mathrm{F} 4$ & $28(13 \%)$ & $181(87 \%)$ & $209(100 \%)$ \\
\hline F5 & $1(5 \%)$ & $20(95 \%)$ & $21(100 \%)$ \\
\hline Total & $30010(88 \%)$ & $4317(12 \%)$ & $34328(100 \%)$ \\
\hline
\end{tabular}

in this paper, we desire a database that extends over a sufficient number of years, and will therefore again consider the 30-yr period 1982-2011.

In Table 2 we give the number of continental USA tornadoes with $L<10 \mathrm{~km}$ and $L \geq 10 \mathrm{~km}$ as a function of Fujita scale intensity for the time period 1982-2011. The total number of "severe" tornadoes (defined as $L \geq 10 \mathrm{~km}$ ) is 4317 (12\% of the database's tornadoes, 1982-2011), with 30010 tornadoes $(L<10 \mathrm{~km})$ omitted. We recognize that a substantial fraction of severe tornadoes (defined as $L \geq 10 \mathrm{~km}$ ) have designation F0 (i.e., $3 \%$ of all F0 tornadoes) and F1 (16\% of all F1 tornadoes), and that a substantial fraction of the alternate definition of severe tornadoes (defined as F2 to F5, $L \geq 0 \mathrm{~km}$ ) have $L<10 \mathrm{~km}$ (e.g., $59 \%$ of all F2 tornadoes, $5 \%$ of all F5 tornadoes). We also note that from the results of Brooks (2004) and this paper (see Fig. 3, Table 1) there is a systematic increase in tornado path lengths as a function of increasing F value. However, there is a large scatter. An important question is whether this scatter can be primarily associated with the damage assessments that give the $\mathrm{F}$ values or whether path lengths are simply not a good measure of tornado intensities.

In order to address this question we return to our comparison between the damage-based Mercalli scale for earth- quakes and the Fujita scale for tornadoes. When a strong earthquake occurs, maps of Mercalli intensities are obtained. These intensities systematically decrease away from the earthquake epicenter, as expected. There are also local variations in values due to local variations in ground shaking intensity. However, in a strong earthquake, hundreds to thousands of Mercalli values are obtained, so that averaging can be carried out to obtain smoothed maps of intensity. These maps are considered useful even if instrumental earthquake magnitudes are available. We will now carry out a systematic study of the statistics of continental USA severe tornadoes, 1982-2011, including further discussion of the two definitions of severe tornadoes, one Fujita scale based, and the other path length based.

\section{Statistics of severe tornadoes}

We first give the dependence of severe tornado (defined as $L \geq 10 \mathrm{~km}$ ) occurrence on time of day, day of the year, and year. In Fig. 4, we give a histogram of times of occurrence of severe tornadoes, 1982-2011. We determine the probability of a severe tornado occurring in a given hour:

$\mathrm{p}(h)=\frac{n_{\mathrm{h}}}{N_{\mathrm{T}}}$ 


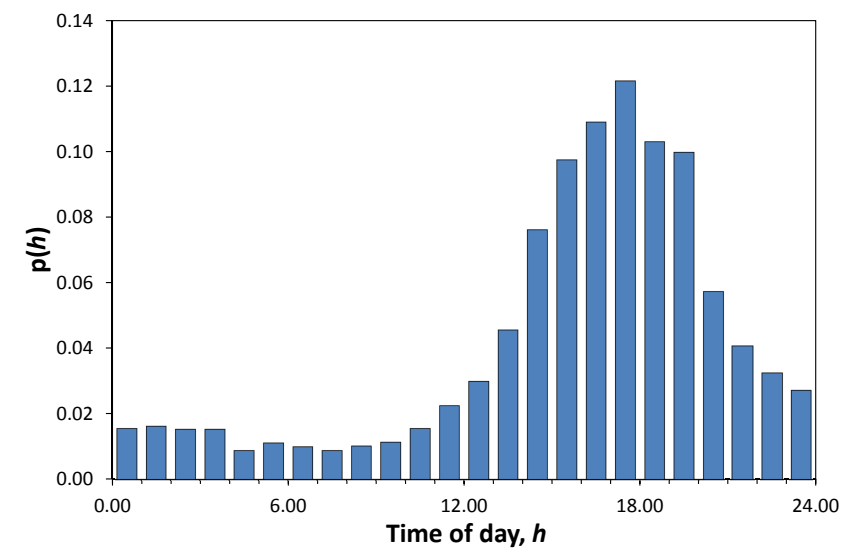

Fig. 4. Histogram of the distribution of continental USA severe tornadoes $(L \geq 10 \mathrm{~km})$ as a function of the hour of the day, $h$ (Central Standard Time). The probabilities $\mathrm{p}(h)$ of a severe tornado occurring are given as a function of $h$ for the time period 1982-2011. Tornado time of day data are from NOAA (2012).

where $n_{\mathrm{h}}$ is the number of severe tornadoes ( $\left.L \geq 10 \mathrm{~km}\right)$ initiated during hour $h$ CST (Central Standard Time), and $N_{\mathrm{T}}$ is the total number of tornadoes $(L \geq 10 \mathrm{~km})$ during the period 1982-2011. The dependence of $\mathrm{p}(h)$ on $h$ is given in Fig. 4. There is an afternoon peak in activity $h=15: 00$ to 20:00 CST. Maximum activity is at $h=17: 00$ to 18:00 CST, with $12 \%$ of all tornadoes initiated during this hour. The results are similar to those given by Kelly et al. (1978) for 17659 tornadoes that occurred between 1950 and 1976.

In Fig. 5, we give the statistics of severe tornado occurrence as a function of day of the year (leap days, 29 February omitted). We use here "convective" days, i.e. the 24-h period from 12:00 UTC of a given day to 12:00 UTC of the following day; this is the same as 06:00-06:00 CST. For each day of the year, 1 to 365 , we give the number of years from 19822011 with at least one severe tornado $L \geq 10 \mathrm{~km}$. There is a peak from April to July (days 91 to 212). The highest peak activity was on day 151 (31 May), with on this day, 15 of the $30 \mathrm{yr}$ having at least one severe tornado.

We next turn to annual variability over the period considered. In Fig. 6, for each year $t=1982$ to 2011 , we give $n_{\mathrm{D}}$ the number of days per year in which one or more severe tornadoes $(L \geq 10 \mathrm{~km})$ occurred. The best-fit linear correlation of this data gives

$n_{\mathrm{D}}=0.280( \pm 0.178) t-510$,

where the uncertainties represent \pm 1 s.e. (standard error) of the slope. The standard error is based on the standard deviation of the $n_{\mathrm{D}}$ values about this trend line, which is 8.3 days. We will use this definition of uncertainty on the slope throughout the paper. On average, the number of days in a year with at least one severe tornado $(L \geq 10 \mathrm{~km})$ increased from $n_{\mathrm{D}}=44$ days in 1982 to 52 days in 2011. The standard error on the slope results in a $95 \%$ confidence in-

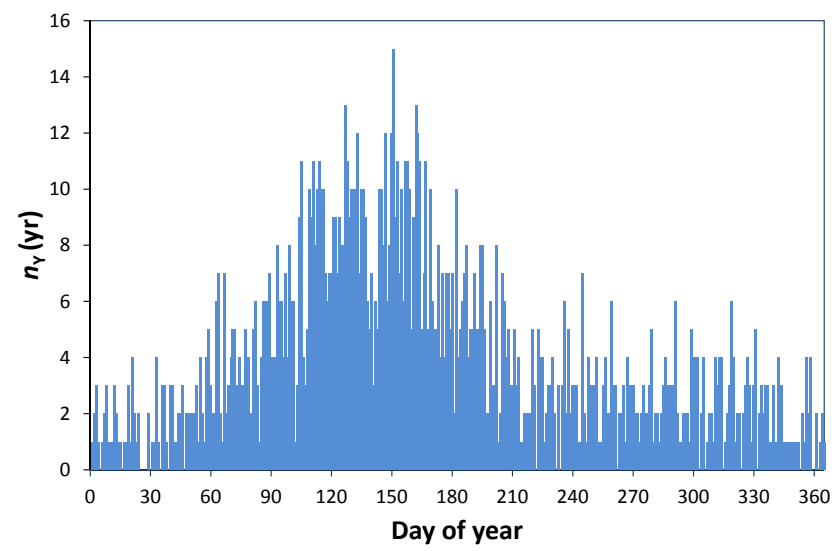

Fig. 5. Distribution of continental USA severe tornadoes $(L \geq 10 \mathrm{~km})$ as a function of day of the year (convective days, 12:00-12:00 UTC, i.e. 06:00-06:00 CST). The number of years $n_{\mathrm{Y}}$ with at least one severe tornado $(L \geq 10 \mathrm{~km})$ is given for each day of the year, 1 to 365 (leap day removed), for the 30-yr period 19822011. Tornado path length data $L$ are from NOAA (2012).

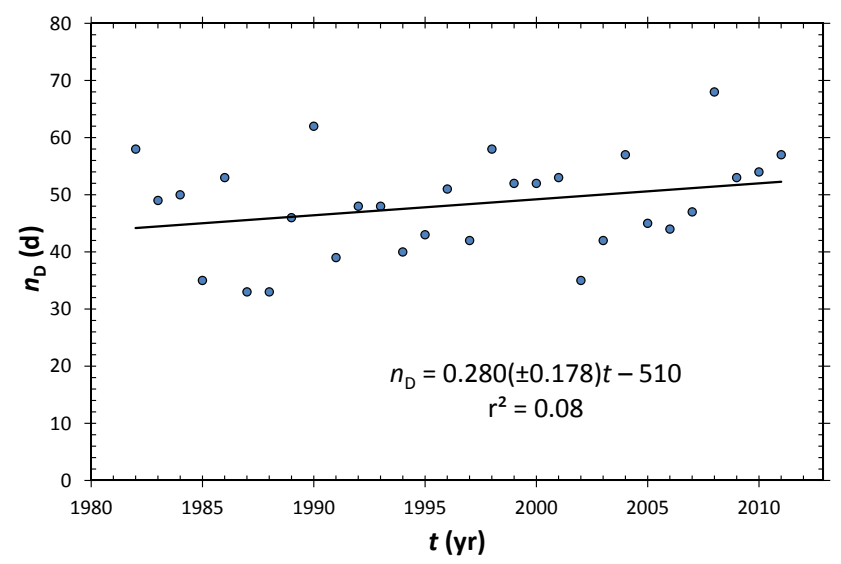

Fig. 6. Number of days per year $n_{\mathrm{D}}$ with at least one continental USA severe tornado (path length $L \geq 10 \mathrm{~km}$ ) is given for the time period 1982-2011. The best-fit linear correlation is also given (Eq. 4), with uncertainties given as \pm 1 s.e. (standard error) of the slope. Tornado path length data $L$ are from NOAA (2012).

terval of $[-0.085,0.645] \mathrm{d} \mathrm{yr}^{-1}$; in other words, considering the scatter of values around the best-fit trend line, there is $95 \%$ confidence that the slope lies somewhere in the range of -0.085 to $0.645 \mathrm{~d} \mathrm{yr}^{-1}$, and therefore a slightly negative or zero trend cannot be rejected.

We next consider the number of severe tornadoes per year in the continental USA for the period 1982-2011. We will utilize two definitions of severe tornadoes, one based on path length exceedance, the other based on Fujita scale intensity exceedance. We first give the annual number of tornadoes per year with path lengths $L \geq 10 \mathrm{~km}$ (and all Fujita scale intensities $\mathrm{F} j, j \geq 0$ ), which we will notate as $N_{\mathrm{Y}[L \geq 10 \mathrm{~km}]}$. These values are given in Fig. 7a, for each year $t=1982$ 


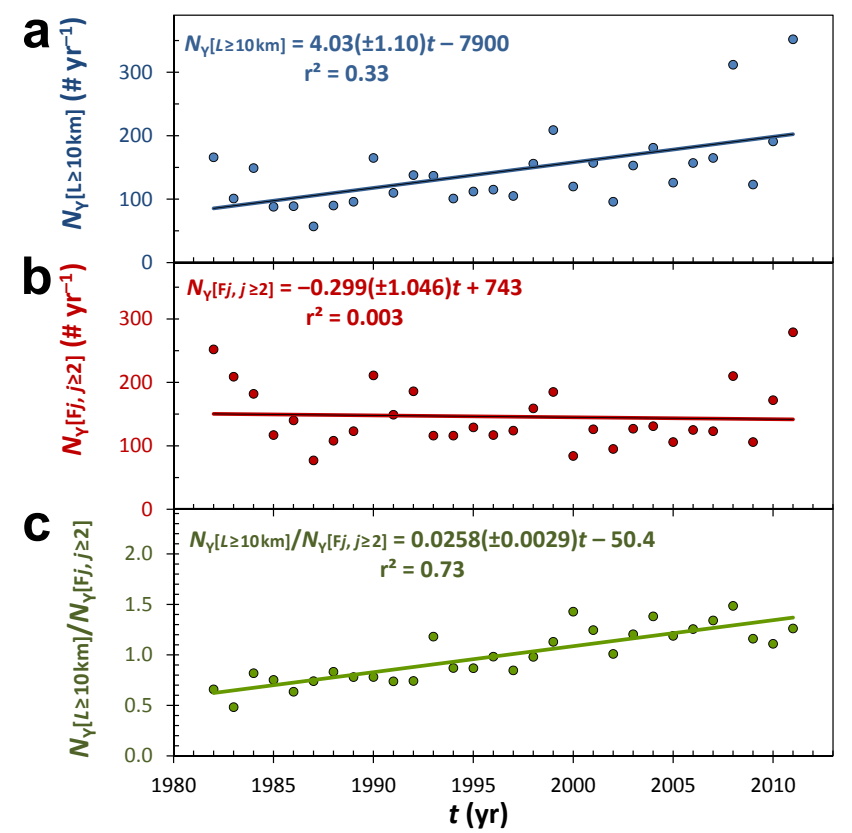

Fig. 7. Continental USA number of severe tornadoes per year, $N_{\mathrm{Y}}$, over the time period 1982-2011, using two definitions for severe tornadoes, one path-length based and the other Fujita scale intensity based. Shown are the total number per year of (a) severe tornadoes $(L \geq 10 \mathrm{~km}), N_{\mathrm{Y}[L \geq 10 \mathrm{~km}]}$ (F0 to F5 considered), (b) severe tornadoes with Fujita (or Enhanced Fujita) scale intensities greater than or equal to $\mathrm{F} 2, N_{\mathrm{Y}[\mathrm{F} j, j \geq 2]}$, (all path lengths $L$ considered). In (c) is shown, per year, the ratio of (a) to (b), i.e. $N_{\mathrm{Y}[L \geq 10 \mathrm{~km}]} / N_{\mathrm{Y}[\mathrm{F} j, j \geq 2]}$. In all three panels, the best-fit linear correlations are shown, with uncertainties given as \pm 1 s.e. (standard error) of the slope. Tornado path length data $L$ are from NOAA (2012).

to 2011. The best-fit linear trend for the annual number of tornadoes is given by:

$N_{\mathrm{Y}[L \geq 10 \mathrm{~km}]}=4.03( \pm 1.10) t-7900$.

In terms of this best-fit, the annual number of tornadoes increased from, on average, $N_{\mathrm{Y}[L \geq 10 \mathrm{~km}]}=87$ tornadoes $\mathrm{yr}^{-1}$ in 1982 to 204 tornadoes $\mathrm{yr}^{-1}$ in 2011 , with the $95 \%$ confidence limits on the slope given by $[1.78,6.28]$ tornadoes $\mathrm{yr}^{-2}$, i.e. within a $95 \%$ confidence, a positive (nonzero) trend is very likely.

As an alternative definition of severe tornadoes, we consider those tornadoes with Fujita scale intensities F2 or larger. Other authors have also considered similar definitions. For example, Verbout et al. (2006) explored the annual variability for tornadoes with $\mathrm{F} j, j \geq 2, j \geq 3, j \geq 4$, for the period 1954-2003. In Fig. 7b we give the annual number of tornadoes per year with Fujita scale intensities $\mathrm{F} j, j \geq 2$ (and all path lengths $L \geq 0 \mathrm{~km}$ ), which we will notate as $\bar{N}_{\mathrm{Y}[\mathrm{F} j, j \geq 2]}$. The best-fit linear trend for the annual number of tornadoes is given by:

$N_{\mathrm{Y}[\mathrm{F} j, j \geq 2]}=-0.299( \pm 1.046) t+743$.

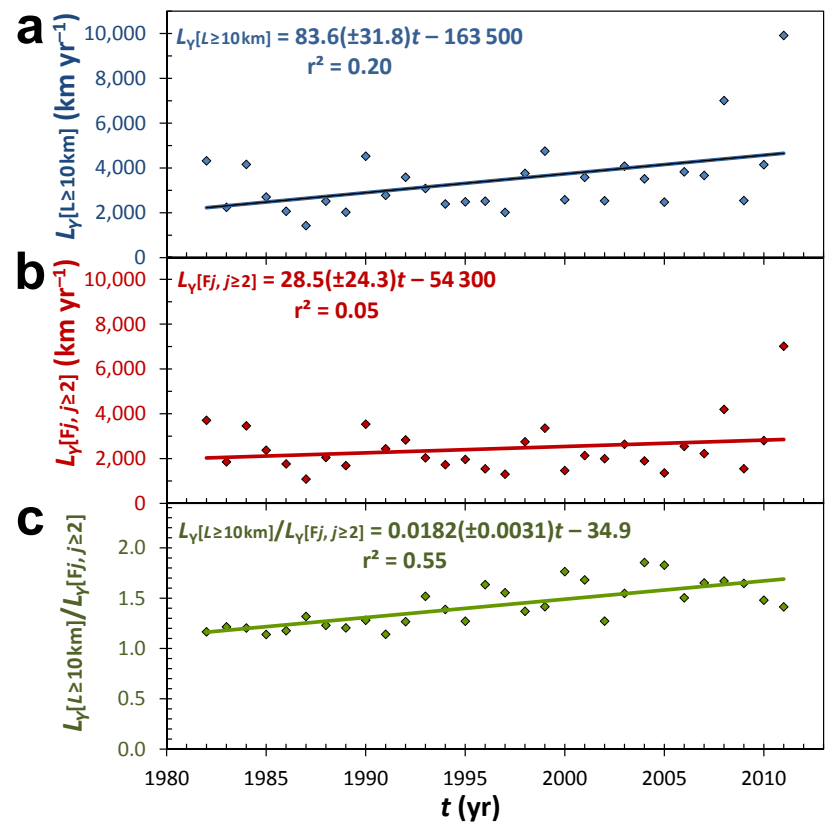

Fig. 8. Continental USA total path length of severe tornadoes per year, $L_{Y}$, over the time period 1982-2011, using two definitions for severe tornadoes, one path-length based and the other Fujita scale intensity based. Shown is the total path length per year for (a) severe tornadoes $(L \geq 10 \mathrm{~km}), L_{Y}[L \geq 10 \mathrm{~km}]$ (F0 to F5 considered), (b) severe tornadoes with Fujita (or Enhanced Fujita) scale intensities greater than or equal to $\mathrm{F} 2, L_{\mathrm{Y}}[\mathrm{F} j, j \geq 2]$, (all path lengths $L$ considered). In (c) is shown, per year, the ratio of (a) to (b), i.e. $L_{\mathrm{Y}[L \geq 10 \mathrm{~km}]} / L_{\mathrm{Y}[\mathrm{F} j, j \geq 2]}$. In all three panels, the best-fit linear correlations are shown, with uncertainties given as \pm 1 s.e. (standard error) of the slope. Tornado path length data $L$ are from NOAA (2012).

In terms of the best-fit, the annual total number of severe tornadoes $(\mathrm{F} j, j \geq 2)$ decreased slightly from, on average, $\quad N_{\mathrm{Y}[\mathrm{F} j, j \geq 2]}=150$ tornadoes $\mathrm{yr}^{-1}$ in 1982 to 139 tornadoes $\mathrm{yr}^{-1}$ in 2011 , but with a large standard error on the slope resulting in a large $95 \%$ confidence interval over which the slope might occur [-2.44, 1.84].

The two methods for defining severe tornadoes have a different dependence on time. To study further this difference, we give the annual ratios $N_{\mathrm{Y}[L \geq 10 \mathrm{~km}]} / N_{\mathrm{Y}[\mathrm{F} j, j \geq 2]}$ in Fig. $7 \mathrm{c}$. The best-fit linear trend to the ratios is given by

$N_{\mathrm{Y}[L \geq 10 \mathrm{~km}]} / N_{\mathrm{Y}[\mathrm{F} j, j \geq 2]}=0.0258( \pm 0.0029) t-50.4$.

In terms of this best-fit, the ratio increases, on average, from 0.73 in 1982 to 1.1 in 2011, with $95 \%$ confidence limits on the slope $[0.020,0.032]$, indicating that within a $95 \%$ confidence, a positive (non-zero) trend is likely.

The increase in deviation between the two measures of the annual numbers of severe tornadoes as a function of year, 1982-2011, appears to be systematic. Before discussing this result we consider, for both definitions of severe tornadoes, the annual total path lengths of severe tornadoes in the 


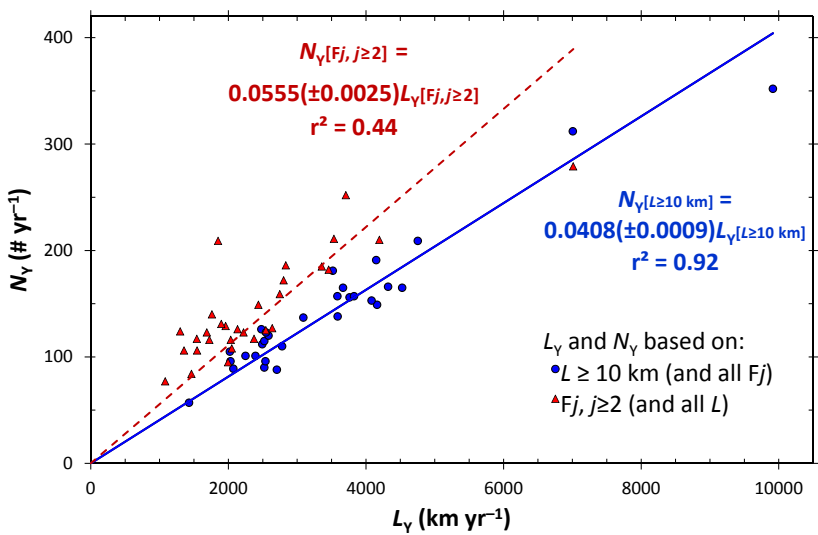

Fig. 9. For continental USA severe tornadoes $(L \geq 10 \mathrm{~km}), 1982-$ 2011 , the number in a given year, $N_{Y}$, is given as a function of the total path length in that year, $L_{Y}$. Results are given for two definitions for severe tornadoes: (i) (blue circles) tornadoes with $L \geq 10 \mathrm{~km}$ (F0 to F5 considered), (ii) (red diamonds) tornadoes with Fujita (or Enhanced Fujita) scale intensities greater than or equal to F2 (all path lengths $L$ considered). The best-fit linear correlations are shown and given in Eqs. (11) and (12), with uncertainties given as \pm 1 s.e. (standard error) of the slope. Tornado path length data $L$ are from NOAA (2012).

continental USA over the period 1982-2011. In Fig. 8a we give for each year $t=1982$ to $2011, L_{Y}[L \geq 10 \mathrm{~km}]$, the annual total path length considering tornadoes with path lengths $L \geq 10 \mathrm{~km}$ (and all Fujita scale intensities $\mathrm{F} j, j \geq 0$ ). The best-fit linear trend for the annual total path length of severe tornadoes $(L \geq 10 \mathrm{~km})$ is given by:

$L_{\mathrm{Y}[L \geq 10 \mathrm{~km}]}=83.6( \pm 31.8) t-163500$.

In terms of the best-fit, the annual total path length of severe tornadoes $(L \geq 10 \mathrm{~km})$ increased from, on average, $L_{\mathrm{Y}[L \geq 10 \mathrm{~km}]}=2700 \mathrm{~km} \mathrm{yr}^{-1}$ in 1982 to $4620 \mathrm{~km} \mathrm{yr}^{-1}$ in 2011. The standard deviation of the values about this trend line is $L_{\mathrm{Y}}[L \geq 10 \mathrm{~km}]=1480 \mathrm{~km} \mathrm{yr}^{-1}$ and the $95 \%$ confidence range on the slope is $[18.4,148.8] \mathrm{km} \mathrm{yr}^{-2}$, in other words, within the $95 \%$ confidence, a positive (non-zero) trend is very likely.

In Fig. 8b we give for each year $t=1982$ to 2011, $L_{\mathrm{Y}[\mathrm{F} j, j \geq 2]}$ the annual total path length considering tornadoes with Fujita scale intensities $\mathrm{F} j, j \geq 2$ (and all path lengths $L \geq 0 \mathrm{~km}$ ). The best-fit linear trend is given by:

$L_{\mathrm{Y}[\mathrm{F} j, j \geq 2]}=28.5( \pm 24.3) t-54300$.

In terms of the best-fit, the annual total path length of severe tornadoes $(\mathrm{F} j, j \geq 2)$ increased from, on average, $L_{\mathrm{Y}[\mathrm{F} j, j \geq 2]}$ $=2190 \mathrm{~km} \mathrm{yr}^{-1}$ in 1982 to $3010 \mathrm{~km} \mathrm{yr}^{-1}$ in 2011. The standard deviation of the values about this trend line is $L_{\mathrm{Y}[\mathrm{F} j, j \geq 2]}=1130 \mathrm{~km} \mathrm{yr}^{-1}$ and the $95 \%$ confidence range on the slope is $[-24.3,78.3] \mathrm{km} \mathrm{yr}^{-2}$; a zero or negative trend cannot be rejected.
The annual total path lengths for both methods increase with time, but the increase is greater for $L_{\mathrm{Y}}[L \geq 10 \mathrm{~km}]$. We again study the differences between the two definitions of severe tornadoes by taking the ratios $L_{\mathrm{Y}[L \geq 10 \mathrm{~km}]} / L_{\mathrm{Y}[\mathrm{F} j, j \geq 2]}$ as shown in Fig. 8c. A best-fit linear trend is given by:

$L_{\mathrm{Y}[L \geq 10 \mathrm{~km}]} / L_{\mathrm{Y}[\mathrm{F} j, j \geq 2]}=0.0182( \pm 0.0031) t-34.9$.

The increase in the annual path length ratios appears systematic, although smaller than the annual number ratios as given in Fig. 7c.

We next study the correlation between the annual numbers of severe tornadoes $N_{\mathrm{Y}}$ and the annual total path lengths $L_{\mathrm{Y}}$. This correlation is illustrated in Fig. 9, where for all severe tornadoes $(L \geq 10 \mathrm{~km})$ from 1982-2011, the annual number $N_{\mathrm{Y}[L \geq 10 \mathrm{~km}]}$ is plotted as a function of the annual total path length $L_{\mathrm{Y}[L \geq 10 \mathrm{~km}]}$ (blue circles). Assuming an intercept of 0 , the best-fit linear correlation is given (Fig. 9) by:

$N_{\mathrm{Y}[L \geq 10 \mathrm{~km}]}=0.0408( \pm 0.0009) L_{\mathrm{Y}[L \geq 10 \mathrm{~km}]}$

with $L_{\mathrm{Y}}[L \geq 10 \mathrm{~km}]$ in $\mathrm{km}$, and relatively little scatter $\left(r^{2}=0.92\right)$. Also shown on Fig. 9 are, for the Fujita-based severe tornado definition $(\mathrm{F} j, j \geq 2)$, the annual number $N_{\mathrm{Y}[\mathrm{F} j, j \geq 2]}$ plotted as a function of the annual total path length $L_{\mathrm{Y}[\mathrm{F} j, j \geq 2]}$ (red triangles). Again, assuming an intercept of 0 , the best-fit linear correlation is given (Fig. 9) by:

$N_{\mathrm{Y}[\mathrm{F} j, j \geq 2]}=0.0555( \pm 0.0025) L_{\mathrm{Y}[\mathrm{F} j, j \geq 2]}$

with $L_{\mathrm{Y}[\mathrm{F} j, j \geq 2]}$ in $\mathrm{km}$, and some scatter $\left(r^{2}=0.44\right)$, a much larger scatter than $N_{\mathrm{Y}[L \geq 10 \mathrm{~km}]}$ vs. $L_{\mathrm{Y}}[L \geq 10 \mathrm{~km}]$ (Eq. 11). It is not unreasonable to expect that as the number of tornadoes increases in a year, so does the total path length of the tornadoes. The relationship shown for the number-length correlations of severe tornadoes will have a tighter linear correlation if the number-length ratio is the same in years of few severe tornadoes and years with many severe tornadoes, i.e. a ratio that is independent of the length considered (scale invariant).

In Fig. 6, we showed that for the number of days per year, $n_{\mathrm{D}}$, where at least one severe tornado with $L \geq 10 \mathrm{~km}$ occurred, there was an $18 \%$ increase over the $30-\mathrm{yr}$ period (1982-2011), but that within the $95 \%$ confidence range of the slope $\left([-0.085,0.645] \mathrm{d} \mathrm{yr}^{-1}\right)$, this trend cannot be considered statistically significant. In Fig. 7, we have given the number of severe tornadoes per year for the period 19822011. We have used two definitions of severe tornadoes, the first based on path lengths $L \geq 10 \mathrm{~km}$ (and all Fujita scale intensities, $\mathrm{F} j, j \geq 0$ ), the second based on Fujita scale considering only those tornadoes with intensities $\mathrm{F} j, j \geq 2$ (and all path lengths $L \geq 0 \mathrm{~km}$ ). The results shown in Fig. 7a and $\mathrm{b}$ show different trends for the two definitions. The lengthbased definition has an increase of $135 \%$ over the $30-\mathrm{yr}$ period and is found to be statistically significant within the 
$95 \%$ confidence limits of the best-fit slope ([1.78, 6.28] tornadoes $\mathrm{yr}^{-2}$ ); whereas, the Fujita scale definition has a decrease of some $7 \%$ and is found not to be statistically significant with the slope's $95 \%$ confidence limits $([-2.44,1.84]$ tornadoes $\mathrm{yr}^{-2}$ ). To study the difference between the two severe tornado definitions, in Fig. 7c, we took the ratio of the values given in Fig. 7a and b, and found a systematic increase in the ratios over time.

In Fig. 8 we have given the annual total path length of severe tornadoes, again using the two definitions for severe tornadoes, for the period 1982-2011. The results given in Fig. 8a (based on path lengths $L \geq 10 \mathrm{~km}$ ) and Fig. 8b (based on Fujita scale intensities $\mathrm{F} j, j \geq 2$ ) again show different trends for the two definitions, but the difference between the trends is smaller than we saw above for the numbers of severe tornadoes per year (Fig. 7). The length-based definition has an increase of $90 \%$ over the 30-yr period and is found to be statistically significant within the $95 \%$ confidence limits of the best-fit slope $\left([18.4,148.8] \mathrm{km} \mathrm{yr}^{-2}\right)$; whereas, the Fujita scale definition has an increase of $37 \%$ and is found not to be statistically significant within the slope's $95 \%$ confidence limits ([-24.3, 78.3] $\left.\mathrm{km} \mathrm{yr}^{-2}\right)$. In Fig. 8c, we took the ratio of the values given in Fig. 8a and b, and again found a systematic increase in the ratios over time.

One possible explanation for the different trends observed over the period 1982-2011 between annual total number and annual total path length for severe tornadoes, is an improvement or change in the surveying. For example, what would have been reported as a single tornado earlier in the period, might later in the period be reported as multiple tornadoes. The annual total path length would not change very much, but the number of tornadoes would increase significantly.

When comparing the two definitions for severe tornadoes, it is important to recognize that for the period considered (1982-2011) only about $50 \%$ of the tornadoes are common to both definitions (i.e., the definitions with criteria based on Fujita scale $\mathrm{F} j, j \geq 2$ vs. path length $L \geq 10 \mathrm{~km}$ ). The Fujitabased severe tornado definition ( $\mathrm{F} j, j \geq 2 ; L \geq 0 \mathrm{~km}$ ) has 4384 tornadoes, of which 2204 have path lengths $\mathrm{L}<10 \mathrm{~km}$ (and thus excluded from the path length definition of severe tornadoes). The path length definition $(L \geq 10 \mathrm{~km}$; all $\mathrm{F} j$, $j \geq 0$ ) has 4317 tornadoes, of which 2137 are F0 and F1 (and thus excluded from the Fujita-based definition of severe tornadoes). It is these differences in tornadoes considered in the two severe tornadoes definitions which result in the different trends observed.

We will use the length-based definition for severe tornadoes ( $L \geq 10 \mathrm{~km}$; all $\mathrm{F} j, j \geq 0)$ in the remainder of this paper for two reasons: (i) We see in Fig. 9 that the proportionality between the annual numbers and path lengths is much more robust for the length-based definition of severe tornadoes compared to the Fujita scale based definition of severe tornadoes. (ii) The focus of this paper will be on path length statistics, thus it is appropriate to define our definition of severe tornadoes using a path length criteria $(L \geq 10 \mathrm{~km}$,

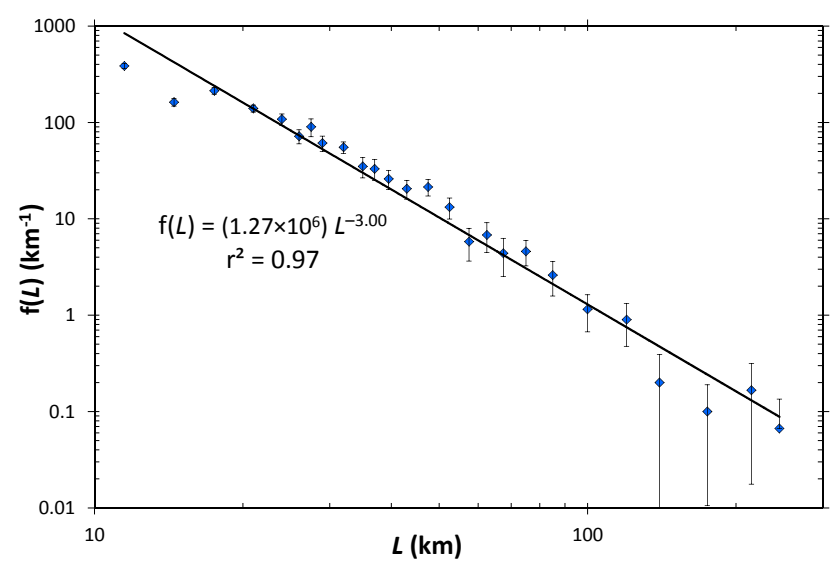

Fig. 10. For continental USA severe tornadoes $(L \geq 10 \mathrm{~km} ; \mathrm{F} j$, $j \geq 0$ ), 1982-2011, the frequency density $\mathrm{f}(L)$ is given as a function of path length $L$. Vertical error bars represent $\pm 2 \sigma$ ( \pm two standard deviations) of the frequency densities $\mathrm{f}(L)$, and are calculated as $\pm(2 \delta N)^{0.5} / \delta L$, where $\delta N$ is the number of tornadoes in a "bin" from $L$ to $L+\delta L$. The $\pm 2 \sigma$ error bars are approximately equivalent to the lower and upper range of the $95 \%$ confidence interval $( \pm 1.96 \sigma)$. The best-fit power-law correlation of the data is also given (Eq. 14). Tornado path length data $L$ are from NOAA (2012).

all $\mathrm{F} j, j \geq 0)$ rather than a Fujita scale criteria $(\mathrm{F} j, j \geq 2$; $L \geq 0 \mathrm{~km}$ ).

We now consider the noncumulative frequency-length statistics of all severe tornadoes $(L \geq 10 \mathrm{~km})$ during the time period 1982-2011. Frequency densities are defined as:

$\mathrm{f}(L)=\frac{\delta N}{\delta L}$,

where $\delta N$ is the number of tornadoes with path lengths between $L$ and $L+\delta L$. In Fig. 10 we plot $\mathrm{f}(L)$ as a function of all $L \geq 10 \mathrm{~km}$, on logarithmic axes, and find a reasonably good power-law correlation:

$\mathrm{f}(L)=1.27 \times 10^{6} L^{-3.00}$,

with $L$ in $\mathrm{km}$. The scaling relationship, Eq. (14), is very good between $20<L<200 \mathrm{~km}$, with some data curvature for $L<20 \mathrm{~km}$. Maximum likelihood analysis was also used to fit a power-law to the original non-binned $L>20 \mathrm{~km}$ data with a power-law exponent found of $-2.93 \pm 0.04$ ( \pm 2 sigma), Kolmogorov-Smirnov $D=0.11$.

We briefly consider the relationship between the cumulative frequency-length data given in Fig. 2 and the noncumulative data given in Fig. 10. The cumulative number $N_{\mathrm{c}}(\geq L)$ is related to the frequency density defined in Eq. (13) by

$N_{\mathrm{c}}=\int_{L}^{\infty} f\left(L^{\prime}\right) \mathrm{d} N$.

Thus $N_{\mathrm{c}}$ is a function of all values of $N(L)$ in the range $L$ to infinity, whereas $f(L)$ is a local measure of the variation of $N(L)$ with $L$ (normalized to "unit" size bins, i.e. 


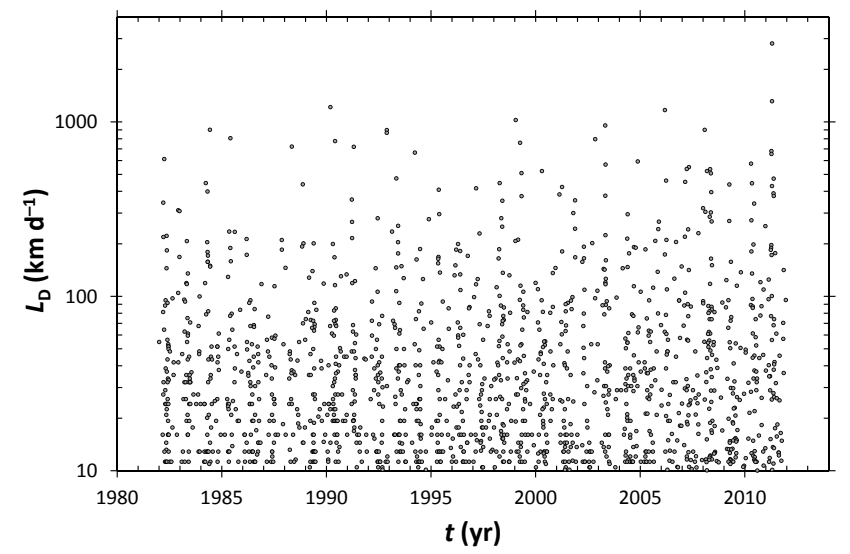

Fig. 11. For continental USA severe tornadoes $(L \geq 10 \mathrm{~km} ; \mathrm{F} j$, $j \geq 0$ ), the total path length, $L_{\mathrm{D}}$, during a convective day (12:0012:00 UTC) is given for the time period 1982-2011. Each $L_{\mathrm{D}}$ represents a quantitative measure of a USA "outbreak" of tornadoes, and is a total of severe tornado path lengths (individual path length data from NOAA, 2012) during a convective day.

$1 \mathrm{~km})$. The rollover for large path length values of $L$ seen in Fig. 2 relative to Fig. 10, can be attributed to a "truncation" of the power-law dependence seen in Fig. 10, for large $L$. The rollover has been shown for several sets of ecological data by Humphries et al. (2010) (see their Fig. 1), and from a theoretical point of view by White et al. (2008).

\section{Statistics of severe tornado outbreaks}

An important aspect of tornado climatology is the occurrence of tornado outbreaks. One definition of a tornado outbreak is the occurrence of multiple tornadoes within a particular synoptic-scale weather system (Glickman, 2000). The NWS SPC database of tornadoes used here (NOAA, 2012) does not explicitly categorize individual tornadoes as part of a specific tornado outbreak. In this paper, we follow the approach of Doswell et al. (2006) and will define a tornado outbreak to include all tornadoes in a convective day (12:00-12:00 UTC) in the continental USA. However, consistent with our studies of individual severe tornadoes, we will consider a severe tornado outbreak to include only those tornadoes with path lengths $L \geq 10 \mathrm{~km}$.

Doswell et al. (2006) considered a variety of measures of the strength of a tornado outbreak based on daily records. They gave the highest weight to the total path length of all tornadoes during a day. In this paper, we will consider the statistics of the total path length, $L_{\mathrm{D}}$, of all severe tornadoes $(L \geq 10 \mathrm{~km})$ in a convective day in the continental USA. In Fig. 11, for 1982-2011, for each convective day that has at least one severe tornado $(L \geq 10 \mathrm{~km})$, we give the daily total path length of tornadoes, $L_{\mathrm{D}}$, for that day. The values for $L_{\mathrm{D}}$ as a function of time appear to be relatively uniform over this time period.

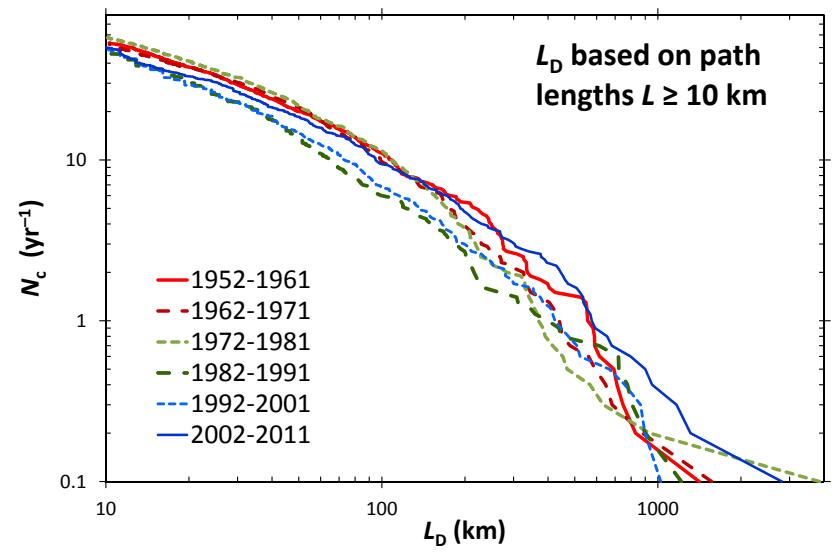

Fig. 12. Cumulative number of continental USA severe tornado outbreaks per year $N_{\mathrm{c}}$ with daily total path lengths greater than or equal to $L_{\mathrm{D}}$, given as a function of $L_{\mathrm{D}}$. Data are given for six 10 -yr periods from 1952-2011. Outbreak path lengths $L_{\mathrm{D}}$ are based only on tornadoes with path lengths $L \geq 10 \mathrm{~km}$ (defined in this paper to be severe tornadoes). Tornado path length data $L$ are from NOAA (2012).

In Fig. 12 we give the cumulative number of severe tornado outbreaks per year $N_{\mathrm{c}}$ with convective daily total path lengths greater than $L_{\mathrm{D}}$, as a function of $L_{\mathrm{D}}$. So that we will be able to compare this with Fig. 1b, values are given for six 10 -yr periods, 1952-2011; only $L \geq 10 \mathrm{~km}$ (severe tornadoes) are used in summing a daily total path length $L_{\mathrm{D}}$. We now compare the daily total path lengths in Fig. 12 with the individual path lengths $(L \geq 10 \mathrm{~km})$ given in Fig. 1b. In Fig. 1b individual curves for the first $30 \mathrm{yr}$ (1952-1981) were consistently higher than for the second $30 \mathrm{yr}$ (1982-2011). This is not the case for the outbreak total path length data $\left(N_{\mathrm{c}}\right.$ vs. $\left.L_{\mathrm{D}}\right)$ shown in Fig. 12 . The daily outbreak $L_{\mathrm{D}}$ data for 1952-1961 and 2002-2011 were generally high; whereas, the data for 1982-1991 and 1992-2001 were generally low. We conclude that although differences in reporting certainly exist, the early data for total lengths of severe tornadoes ( $L \geq 10 \mathrm{~km}$ ) during a convective day were more robust than the early data for tornado path lengths taken individually.

We next make a rough estimate of the risk of severe tornado outbreaks (daily total path length $L_{\mathrm{D}}$ ) in analogy to our estimate for the risk of individual tornadoes (individual path lengths $L$ ) given in Fig. 2. In Fig. 13 we give the outbreaks per year $N_{\mathrm{c}}$ with convective daily total path lengths greater than $L_{\mathrm{D}}$, as a function of $L_{\mathrm{D}}$. Consistent with the discussion given above (and Fig. 2) for the purposes of this estimate, we use all data from 1952-2011. During this 60yr period, the longest daily total path length $L_{\mathrm{D}}=3852 \mathrm{~km}$ occurred on 3 April 1974 and included 105 tornadoes with $L \geq 10 \mathrm{~km}$, the 2nd longest $L_{\mathrm{D}}=2815 \mathrm{~km}$ on 27 April 2011, and the 3rd longest $L_{\mathrm{D}}=1566 \mathrm{~km}$ on 11 April 1965. The 4th and 5th longest daily outbreaks also occurred in the 


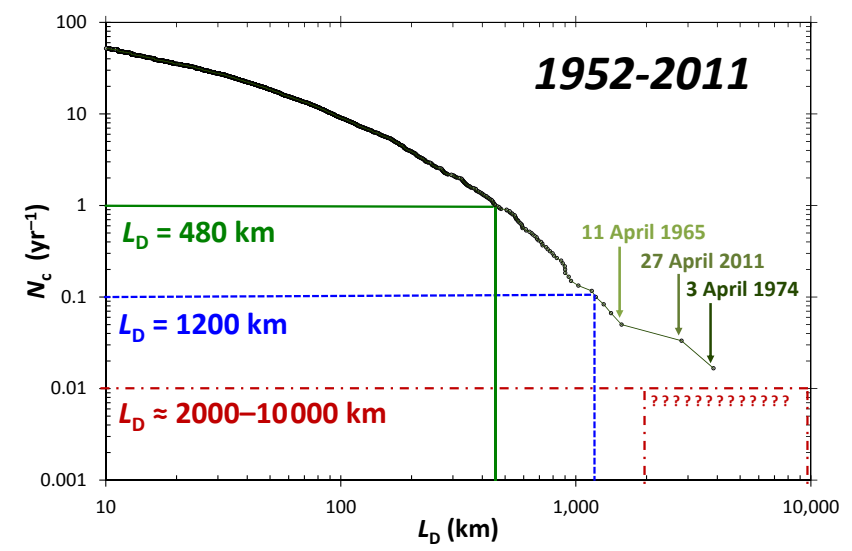

Fig. 13. Cumulative number of continental USA severe tornado outbreaks per year $N_{\mathrm{c}}$ with daily total path lengths greater than or equal to $L_{\mathrm{D}}$, given as a function of $L_{\mathrm{D}}$. The data are for the period 1952 2011 with outbreak total path lengths $L_{\mathrm{D}}$ based only on tornadoes with path lengths $L \geq 10 \mathrm{~km}$ (defined in this paper to be severe tornadoes). The three longest outbreaks are identified by vertical arrows. Using this data, rough estimates are made for the length of the expected 1-yr, 10-yr, and 100-yr outbreaks. Tornado path length data $L$ are from NOAA (2012).

month of April (30 April 1954, $L_{\mathrm{D}}=1412 \mathrm{~km} ; 26$ April $\left.2011, L_{\mathrm{D}}=1313 \mathrm{~km}\right)$.

We use the data in Fig. 13 to give a rough estimate of the hazard of severe tornado outbreaks and estimate the annual tornado outbreak to have a daily path length of $L_{\mathrm{D}} \geq 480 \mathrm{~km}$. On average, not taking into account any changing trends over time, we would expect in any given year an outbreak with daily path length of $L_{\mathrm{D}} \geq 480 \mathrm{~km}$. The ten year tornado outbreak (the longest path length or greater expected, on average, in a $10-\mathrm{yr}$ period) is $L_{\mathrm{D}} \geq 1200 \mathrm{~km}$. An extrapolation of the curve is (similar to Fig. 2) difficult for longer recurrence periods, as the shape of the statistical distribution for the largest lengths is again unclear. One estimate is that the 100 -yr tornado (the longest path length or greater expected in a 100 -yr period) is in the range of $2000-10000 \mathrm{~km}$. This estimate has a large uncertainty as the extrapolation of the data is uncertain for these extreme values. It is interesting to note that in the 60-yr period from 1952-2011 we had two tornado outbreaks with $L_{\mathrm{D}}>2800 \mathrm{~km}$.

As just discussed above, we believe that over the period 1952-2011, total convective day lengths of severe tornadoes $(L \geq 10 \mathrm{~km})$ are relatively robust when comparing earlier decades with later decades in terms of data quality. However, for the remainder of the analyses of this section, as some differences do exist between the earlier and the later decades, and so that we are consistent with earlier sections in this paper, we will return to considering only the period 1982-2011.

We now consider (Fig. 14) for the period 1982-2011 the correlation between $N_{\mathrm{D}}$ the total number of severe tornadoes $(L \geq 10 \mathrm{~km})$ in a convective day (i.e., a continental USA "outbreak") and $\overline{L_{\mathrm{D}}}$ the mean of the convective daily total

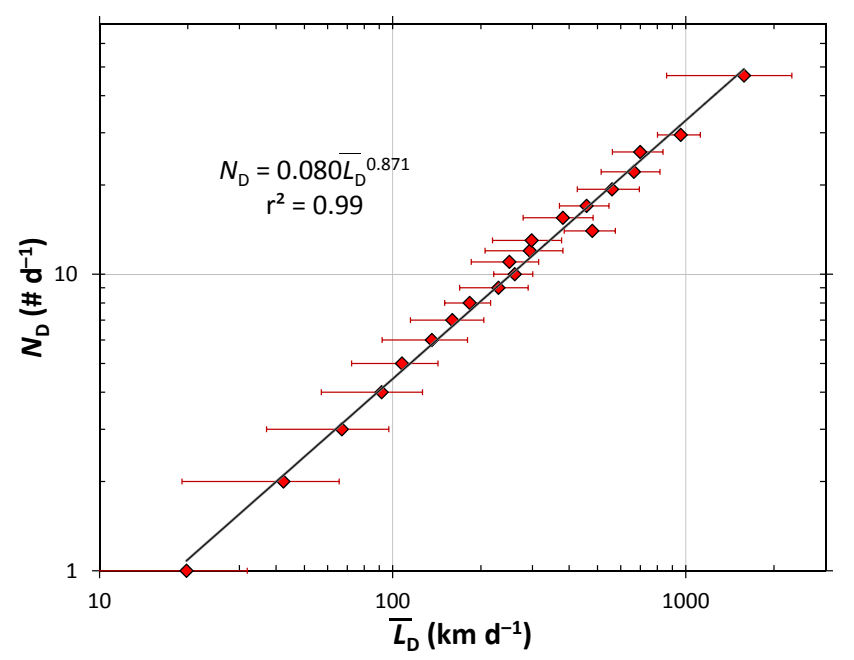

Fig. 14. The total number of severe tornadoes $(L \geq 10 \mathrm{~km})$ in a continental USA “outbreak" $N_{\mathrm{D}}$ during the period (1982-2011), is given as a function of $\overline{L_{\mathrm{D}}}$ the mean of the convective daily total tornado path lengths for all days where $N_{\mathrm{D}}$ is the same value. Daily values are for convective days (12:00-12:00 UTC). Horizontal error bars represent $\pm 1 \sigma$ ( \pm one standard deviation) of the $L_{\mathrm{D}}$ for a given $N_{\mathrm{D}}$. The best-fit power-law correlation of the data is also given (Eq. 16). Tornado path length data $L$ are from NOAA (2012).

tornado path lengths for all days where $N_{\mathrm{D}}$ is the same value. We also consider the standard deviation of $L_{\mathrm{D}}$ for each $N_{\mathrm{D}}$. For example, there are 79 days where $N_{\mathrm{D}}=4$ severe tornadoes occur during the day; the mean ( \pm standard deviation) of the total tornado daily path lengths $L_{\mathrm{D}}$ for those 74 occurrences is $\overline{L_{\mathrm{D}}}=91.7( \pm 34.7) \mathrm{km}$. Because there are relatively few outbreaks with large values of $N_{\mathrm{D}}$, we consider the mean of all $L_{\mathrm{D}}$ over multiple values of $N_{\mathrm{D}}$. The best-fit linear correlation to $N_{\mathrm{D}}$ as a function of $\overline{L_{\mathrm{D}}}$ (Fig. 14) is a power-law relationship:

$N_{\mathrm{D}}=0.080\left(\overline{L_{\mathrm{D}}}\right)^{0.871}$

over the range $20<\overline{L_{\mathrm{D}}}<1600 \mathrm{~km} \mathrm{~d}^{-1}$. This power-law correlation is quite robust as it extends over almost two orders of magnitude. With a power-law exponent of 0.87 , the correlation between the number of severe tornadoes in a daily USA outbreak, $N_{\mathrm{D}}$, and the mean daily total tornado path length, $\bar{L}_{\mathrm{D}}$, is almost linear (i.e., exponent 1.0). We conclude that $N_{\mathrm{D}}$ and $\overline{L_{\mathrm{D}}}$ (calculated for all tornadoes $L \geq 10 \mathrm{~km}$ ) are equivalent measures of the strength of a USA severe tornado outbreak.

We next give the frequency-length statistics of daily USA tornado outbreaks for the time period 1982-2011. Similar to the definition of the frequency-density function $f(L)$ given in Eq. (13), we plot $f\left(L_{\mathrm{D}}\right)$ vs. $L_{\mathrm{D}}$ in Fig. 15 on logarithmic axes, and find an excellent power-law correlation:

$\mathrm{f}\left(L_{\mathrm{D}}\right)=8325 L_{\mathrm{D}}{ }^{-1.81}$ 


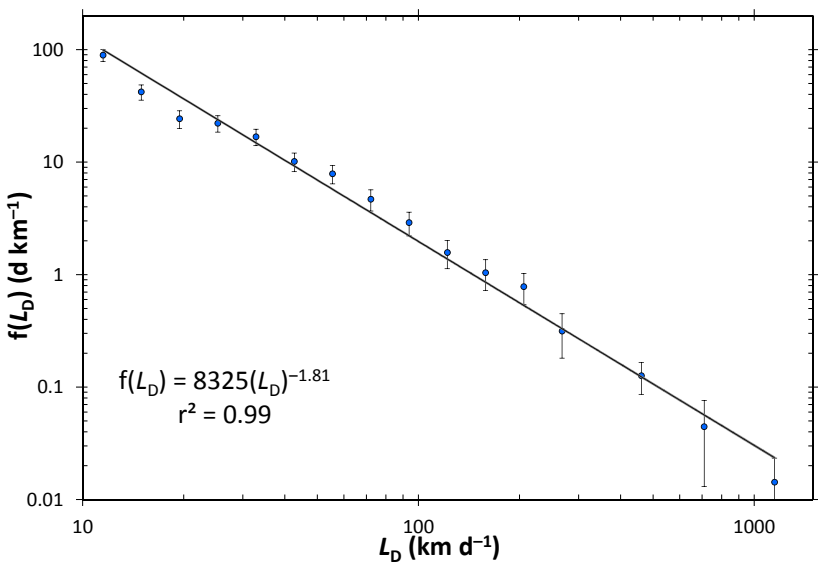

Fig. 15. The frequency-length statistics of continental USA daily tornado outbreaks during the period 1982-2011. The frequency densities $\mathrm{f}\left(L_{\mathrm{D}}\right)$ are given as a function of $L_{\mathrm{D}}$, the total path length of all severe tornadoes $(L \geq 10 \mathrm{~km})$ during a USA daily outbreak. Daily values are for convective days (12:00-12:00 UTC). Vertical error bars represent $\pm 2 \sigma$ ( \pm two standard deviations) and calculated as given in Fig. 10 caption. The best-fit power-law correlation of the data is also given (Eq. 17). Tornado path length data $L$ are from NOAA (2012)

with $L_{\mathrm{D}}$ in $\mathrm{km} \mathrm{d}^{-1}$. This power-law relationship is found to be robust over about two orders of magnitude, $10<L_{\mathrm{D}}<1000 \mathrm{~km} \mathrm{~d}^{-1}$. Maximum likelihood analysis was also used to fit a power-law to the original non-binned $L_{\mathrm{D}}$ data, with a power-law exponent found of $-1.76 \pm 0.03$ ( \pm 2 sigma), Kolmogorov-Smirnov $D=0.10$. The cumulative frequency-length data given in Fig. 13 for $N_{\mathrm{c}}\left(\geq L_{\mathrm{D}}\right)$ has a "rollover" for large $L_{\mathrm{D}}$, compared to the noncumulative data given in Fig. 15. The explanation given at the end of Sect. 3 for cumulative vs. noncumulative statistics of severe individual tornado path length statistics $L$, is also applicable to the outbreak data $L_{\mathrm{D}}$.

As our final study of tornado statistics, we will consider the distribution of tornado path lengths during a severe tornado outbreak. For this purpose, we consider two different sized outbreaks, a very large outbreak on 27 April 2011 with 67 severe tornadoes (total path length $L_{\mathrm{D}}=2816 \mathrm{~km}$ ) and a smaller outbreak on 25 May 2011 with 16 severe tornadoes (total path length $L_{\mathrm{D}}=376 \mathrm{~km}$ ). As both severe tornado outbreaks are chosen from 2011 records, we believe that the path length records should be very robust with respect to completeness and quality of the data. The outbreak on 27 April 2011 was the 2nd largest continental USA outbreak during the period 1952-2011 (Fig. 13), the 25 May 2011 the 80th largest. In Fig. 16 we give the cumulative number of tornadoes $N_{\mathrm{c}}$ with path lengths $L \geq 10 \mathrm{~km}$, as a function of $L$. The longest individual path length for 27 April 2011 was $L=212.4 \mathrm{~km}$ and for 25 May $2011 L=76.3 \mathrm{~km}$. In both the large and medium convective day outbreak, there is a similar and systematic distribution of severe tornado path lengths,

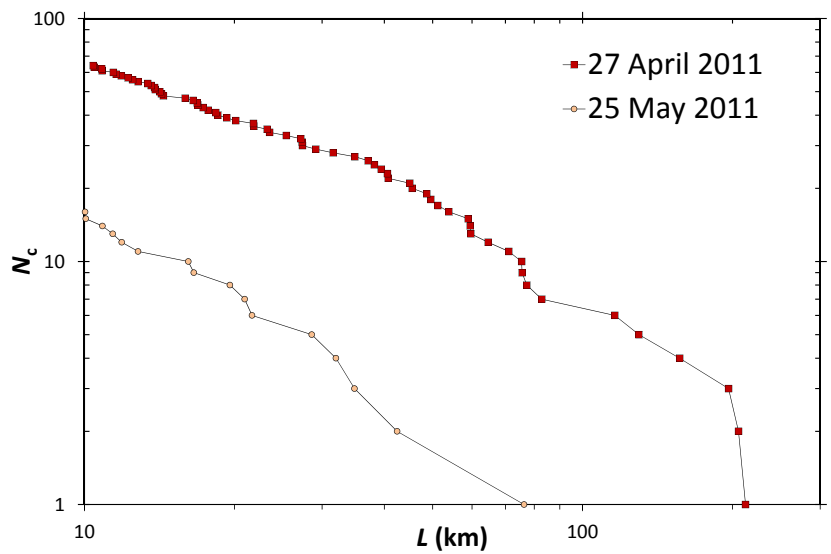

Fig. 16. Distribution of severe tornado $(L \geq 10 \mathrm{~km})$ path lengths during two convective day (12:00-12:00 UTC) outbreaks in the continental USA. The cumulative number of severe tornadoes $N_{\mathrm{c}}$ with path lengths greater than or equal to $L$, given as a function of $L$. Results are given for outbreaks on the 27 April 2011 (67 severe tornadoes) and 25 May 2011 (16 severe tornadoes). Tornado path length data $L$ are from NOAA (2012).

with similar scaling. The examples given in Fig. 16 show that tornado outbreaks appear to have robust distributions of severe tornado intensities as given by path lengths.

\section{Discussion and conclusions}

In any study of the statistics of a natural hazard it is necessary to have a reliable database. In the case of tornadoes, an important question is what a database should contain. The standard measure of tornado intensity is the damage-based Fujita scale. The only other widely available measure of tornado intensity is the path length of touchdown caused by a tornado.

To aid the reader, in Table 3 we give a summary of the symbols and abbreviations that we have used in this paper. In Fig. 1, we have given the cumulative number of tornadoes per year with path lengths greater than $L$. The data are given for six 10-yr periods, between 1952-2011. The data during the three 10-yr periods, 1982-2011, are relatively consistent and differ substantially from earlier periods. This difference can be attributed to systematic NWS tornado surveys introduced in the early 1980s. Based on Fig. 1's data, we restrict our statistical studies of individual tornado path lengths $L$ to the period 1982-2011. In Fig. 2, we gave cumulative-path length statistics $\left(N_{\mathrm{c}} \geq L\right)$ for the entire period 1982-2011 and $L \geq 10 \mathrm{~km}$. We used this to make a rough estimate for the longest tornado path length (or greater) expected, on average, every 1,10,100 yr, giving values (respectively) of 115 , 215 and $280-500 \mathrm{~km}$. The use of these frequency-size statistics to calculate the probability of given path length tornadoes occurring, implicitly assumes weak stationarity of the severe tornado time series. We acknowledge that there exists 
Table 3. List of symbols and abbreviations.

\begin{tabular}{|c|c|c|}
\hline Variable & Units & Description \\
\hline$\delta N$ & \# & The number of tornadoes with path lengths between $L$ and $L+\delta L$. \\
\hline$\sigma$ & & Standard deviation. \\
\hline $\mathrm{f}(L), \mathrm{f}\left(L_{\mathrm{D}}\right)$ & varies & Frequency density of $L$ (see Eq. 13 ) or $L_{\mathrm{D}}$. \\
\hline $\mathrm{F} j$ & & Fujita scale intensities, $\mathrm{F} j, j=0,1,2, \ldots, 5$. \\
\hline$h$ & & Hour of the day. \\
\hline$j$ & & $\begin{array}{l}\text { Variable representing the Fujita scale intensities, where } \mathrm{F} 0, \mathrm{~F} 1, \mathrm{~F} 2, \mathrm{~F} 3, \mathrm{~F} 4, \mathrm{~F} 5 \text { are given as } \mathrm{F} j \text {, } \\
j=0,1,2, \ldots, 5 \text {. }\end{array}$ \\
\hline$L$ & $\mathrm{~km}$ & Individual tornado touchdown path length. \\
\hline$L_{\mathrm{D}}$ & $\mathrm{km} \mathrm{d}^{-1}$ & $\begin{array}{l}\text { Total touchdown path length of severe tornadoes (path lengths } L \geq 10 \mathrm{~km} \text {; all Fujita scale intensities } \mathrm{F} j \text {, } \\
j \geq 0 \text { ) in a convective day (12:00-12:00 UTC). }\end{array}$ \\
\hline$\overline{L_{\mathrm{D}}}$ & $\mathrm{km} \mathrm{d}^{-1}$ & $\begin{array}{l}\text { Mean of the convective daily }(12: 00-12: 00 \text { UTC) total path lengths of severe tornadoes (path lengths } \\
L \geq 10 \mathrm{~km} \text {; all Fujita scale intensities } \mathrm{F} j, j \geq 0) \text { over multiple days. }\end{array}$ \\
\hline$\overline{L_{\mathrm{F} j}}$ & $\mathrm{~km}$ & Mean of all tornado path lengths $L$ at given Fujita scale intensities $\mathrm{F} j, j=0,1,2, \ldots, 5$. \\
\hline$L_{Y}$ & $\mathrm{~km} \mathrm{yr}^{-1}$ & $\begin{array}{l}\text { Total path length of severe tornadoes in a year (see } L_{\mathrm{Y}[\mathrm{F} j, j \geq 2]} \text { and } L_{\mathrm{Y}}[L \geq 10 \mathrm{~km}] \\
\text { definitions of severe tornadoes). }\end{array}$ \\
\hline$L_{\mathrm{Y}}[\mathrm{F} j, j \geq 2]$ & $\mathrm{km} \mathrm{yr}^{-1}$ & $\begin{array}{l}\text { Total path length of severe tornadoes (defined as Fujita scale intensities } \mathrm{F} j, j \geq 2 \text { and all } L \geq 0 \mathrm{~km} \text { ) in a } \\
\text { year. }\end{array}$ \\
\hline$L_{\mathrm{Y}}[L \geq 10 \mathrm{~km}]$ & $\mathrm{km} \mathrm{yr}^{-1}$ & $\begin{array}{l}\text { Total path length of severe tornadoes (defined as path lengths } L \geq 10 \mathrm{~km} \text { and all Fujita scale intensities } \\
\mathrm{F} j, j \geq 0 \text { ) in a year. }\end{array}$ \\
\hline$N_{\mathrm{c}}$ & \# & $\begin{array}{l}\text { Cumulative number of: (i) tornadoes with path lengths greater than or equal to } L \text {; (ii) outbreaks with total } \\
\text { path lengths in a convective day greater than or equal to } L_{\mathrm{D}} \text {. }\end{array}$ \\
\hline$n_{\mathrm{D}}$ & $\mathrm{d}$ & $\begin{array}{l}\text { Number of days per year with at least one severe tornado (path lengths } L \geq 10 \mathrm{~km} \text {; all Fujita scale } \\
\text { intensities } \mathrm{F} j, j \geq 0 \text { ). }\end{array}$ \\
\hline$N_{\mathrm{D}}$ & $\# d^{-1}$ & $\begin{array}{l}\text { Total number of severe tornadoes (path lengths } L \geq 10 \mathrm{~km} \text {; all Fujita scale intensities } \mathrm{F} j, j \geq 0 \text { ) in a } \\
\text { convective day (12:00-12:00 UTC). }\end{array}$ \\
\hline$n_{\mathrm{h}}$ & \# & $\begin{array}{l}\text { Total number of severe tornadoes (path lengths } L \geq 10 \mathrm{~km} \text {; all Fujita scale intensities } \mathrm{F} j, j \geq 0 \text { ) initiated } \\
\text { during hour, } h \text {. }\end{array}$ \\
\hline$N_{\mathrm{T}}$ & \# & Total number of values in the dataset considered. \\
\hline$n_{Y}$ & $\mathrm{yr}$ & $\begin{array}{l}\text { Number of "years per day of the year", with at least one severe tornado (path lengths } L \geq 10 \mathrm{~km} \text {; all } \\
\text { Fujita scale intensities } \mathrm{F} j, j \geq 0 \text { ). }\end{array}$ \\
\hline$N_{\mathrm{Y}}$ & $\# \mathrm{yr}^{-1}$ & $\begin{array}{l}\text { Total number of severe tornadoes in a year (see } N_{\mathrm{Y}[\mathrm{F} j, j \geq 2]} \text { and } N_{\mathrm{Y}[L \geq 10 \mathrm{~km}]} \text { for two different definitions } \\
\text { of severe tornadoes). }\end{array}$ \\
\hline$N_{\mathrm{Y}[\mathrm{F} j, j \geq 2]}$ & $\# \mathrm{yr}^{-1}$ & Total number of severe tornadoes (defined as Fujita scale intensities $\mathrm{F} j, j \geq 2$ and all $L \geq 0 \mathrm{~km}$ ) in a year. \\
\hline$N_{\mathrm{Y}}[L \geq 10 \mathrm{~km}]$ & $\# \mathrm{yr}^{-1}$ & $\begin{array}{l}\text { Total number of severe tornadoes (defined as path lengths } L \geq 10 \mathrm{~km} \text { and all Fujita scale intensities } \mathrm{F} j \text {, } \\
j \geq 0 \text { ) in a year. }\end{array}$ \\
\hline $\mathrm{p}(h)$ & & $\begin{array}{l}\text { Probability of a severe tornado (path lengths } L \geq 10 \mathrm{~km} \text {; all Fujita scale intensities F } j, j \geq 0 \text { ) occurring } \\
\text { for a given hour of the day, } h \text {. }\end{array}$ \\
\hline s.e. & & Standard error. \\
\hline$t$ & $\mathrm{yr}$ & Time in years. \\
\hline UTC & & Coordinated Universal Time \\
\hline WPM & & Warning Preparedness Meteorologist \\
\hline
\end{tabular}

an annual seasonality within the time series, and a clustering of values for tornadoes that occur with given atmospheric conditions.

The basic purpose of this paper has been to consider the statistics of tornado touchdown path lengths as a measure of tornado intensity. Since the standard measure of tornado intensity in the USA is the Fujita scale, we consider the variability of path lengths $L$ for a specified Fujita scale intensity, $\mathrm{F} j, j=0,1,2, \ldots, 5$. This dependence for our period of study, 1982-2011, was given in Fig. 3. Although there is a systematic increase in mean path length with increasing
Fujita scale intensity, there is also a large variability. A reasonably good scaling of the mean touchdown path lengths as given in Eq. (1) was found for strong (F2, F3) and violent $(\mathrm{F} 4, \mathrm{~F} 5)$ tornadoes. The deviation from this scaling for weak (F0, F1) tornadoes is likely due to limitations of the Fujita scale for weak tornadoes and/or measurement problems with determining path lengths for these weak tornadoes.

Over the period 1982-2011, we have given (Fig. 6) the annual number of days $n_{\mathrm{D}}$ during which at least one severe (defined as $L \geq 10 \mathrm{~km}$ ) tornado occurred and (Figs. 7 and 8), for two different definitions of severe tornadoes, the annual 
total number $N_{\mathrm{Y}}$ and annual total path lengths $L_{\mathrm{Y}}$ of severe tornadoes. The two definitions of severe tornadoes included: (i) path length-based ( $L \geq 10 \mathrm{~km}$; all $\mathrm{F} j, j \geq 0$ ) with 4317 severe tornadoes; (ii) Fujita-based (Fj, $j \geq 2$; all $L$ ) with 4384 severe tornadoes. However, only about half of the severe tornadoes are included in both definitions. Although in most cases systematic increases over the period 1982-2011 were observed, there was also considerable scatter. Only for annual total number and path length $\left(N_{\mathrm{Y}}\right.$ and $\left.L_{\mathrm{Y}}\right)$, using the path-length definition of severe tornadoes, was the increase significant within the lower and upper limits of the $95 \%$ confidence limits on the slope. We note that this trend for these values is only for the 30-yr period 1982-2011, and that extrapolating forward or backwards in time, will not necessarily have the same positive trend.

In Fig. 9, we gave the total number of severe tornadoes in a year, $N_{Y}$, as a function of the total path length of tornadoes in that year, $L_{Y}$, for both definitions of severe tornadoes. We observed that the correlations are much more robust (using a linear correlation) for the path-length definition ( $L \geq 10 \mathrm{~km}$; all $\mathrm{F} j, j \geq 0$ ) than the Fujita scale definition ( $\mathrm{F} j, j \geq 2$; all $L \geq 0 \mathrm{~km}$ ). We then argued the use of the length-based definition for severe tornadoes ( $L \geq 10 \mathrm{~km}$; all $\mathrm{F} j, j \geq 0$ ) in the remainder of the paper based on Fig. 9's more robust behaviour for the length-based definition and also the paper's focus on path length statistics. We therefore used this database of 4317 severe continental USA tornadoes ( $L \geq 10 \mathrm{~km}$ ) that occurred over the time period 1982-2011.

There is no question that Fujita scale evaluations of tornado intensities are very useful. However, results in this paper, along with other work, would indicate that tornado touchdown path lengths may also be a very useful measure of the intensity of both individual tornadoes and tornado outbreaks. We believe that the definition provided here of a severe tornado ( $L \geq 10 \mathrm{~km}$; all $\mathrm{F} j, j \geq 0$ ), is easily quantifiable and provides robust statistics.

In Fig. 10, we have given the dependence of the frequency density of severe tornado path lengths $L \geq 10 \mathrm{~km}$ on path length $L$. The frequency density gives a local measure of path length scaling. Over the touchdown path length range $20<L<200 \mathrm{~km}$, we found reasonably good powerlaw scaling (Eq. 14) of the frequency density as a function of $L$, with power-law exponent about -3.00 .

Tornado outbreaks are an important feature of tornado climatology. Ideally, a tornado outbreak would be associated with a particular synoptic-scale weather system. Although location information is available for each tornado path length, the association of specific tornadoes with a specified outbreak are still difficult to make in a rapid and systematic way. We follow the approach used by Doswell et al. (2006) who defines a tornado outbreak to be all tornadoes in a $24-\mathrm{h}$ period in the continental USA, where the 24-h period is a convective day (12:00-12:00 UTC, i.e. 06:0006:00 CST). Consistent with our study of severe individual tornadoes with path lengths $L \geq 10 \mathrm{~km}$, we define a severe tornado outbreak to be all severe tornadoes $(L \geq 10 \mathrm{~km})$ during a convective day in the continental USA. As two measures of severe outbreak intensity, we utilize the number of severe tornadoes during a convective day, $N_{\mathrm{D}}$, and the total path length of severe tornadoes during a convective day, $L_{\mathrm{D}}$.

In Fig. 12, we gave the cumulative number of severe tornado outbreaks per year $N_{\mathrm{c}}$ with daily total path lengths greater than $L_{\mathrm{D}}$, as a function of $L_{\mathrm{D}}$. In analogy to Fig. 1, we do this for six 10-yr periods between 1952-2011. In Fig. 1, the individual path length statistics $\left(N_{\mathrm{c}}\right.$ vs. $\left.L\right)$ for the first three decade periods (1952-1981) were consistently higher than the second three decades (1982-2011). This is not the case for the outbreak daily total path length data $\left(N_{\mathrm{c}}\right.$ vs. $\left.L_{\mathrm{D}}\right)$ given in Fig. 12, where the six decades do not appear to be biased by being earlier or later in the 60 year period considered. For this reason, we considered next, in Fig. 13, the severe outbreak cumulative path length statistics for (in analogy to Fig. 2) the entire period 1952-2011. During this period, the most extreme convective day outbreak was on 3 April 1974 , with $L_{\mathrm{D}}=3852 \mathrm{~km}$, and the second most extreme on 27 April 2011 with $L_{\mathrm{D}}=2815 \mathrm{~km}$. The data in Fig. 13 was used to make a rough estimate for the length (or greater) of a severe outbreak's convective day path length expected, on average, every 1, 10, $100 \mathrm{yr}$, giving values (respectively) of 480,1200 and $2000-10000 \mathrm{~km}$.

In Fig. 14, we found an excellent, near-linear relationship, between the number of severe tornadoes $(L \geq 10 \mathrm{~km})$ in a given outbreak $N_{\mathrm{D}}$, and the mean of the total convective day path lengths $\overline{L_{\mathrm{D}}}$ corresponding to outbreaks with that number $N_{\mathrm{D}}$. This relationship is the same for severe tornado outbreaks with many tornadoes and also with very few tornadoes. In Fig. 15, we gave the dependence of the frequency density of severe tornado outbreaks as a function of the total convective day path lengths, $L_{\mathrm{D}}$. Over the range $10<L_{\mathrm{D}}<1000 \mathrm{~km}$, we found reasonably good power-law scaling (Eq. 17) of the frequency density as a function of $L_{\mathrm{D}}$, with power-law exponent about -1.8 . This approximate scaling is evidence for a degree of self-organization in the statistical occurrence of severe tornado outbreaks.

In addition to our studies of the distributions of path lengths of individual tornadoes and convective day total path lengths of severe tornado outbreaks, we have also studied the distribution of path lengths during two individual severe tornado $(L \geq 10 \mathrm{~km})$ outbreaks that occurred during the year 2011: 27 April 2011 (67 severe tornadoes) and 25 May 2011 (16 severe tornadoes). In Fig. 16 we gave, separately for the two severe outbreak days, the cumulative number of severe tornadoes $(L \geq 10 \mathrm{~km})$ with path lengths greater than $L$ as a function of $L$. An approximate scaling was observed indicating again, statistical self-organization during the tornado outbreak itself of the path lengths.

Based on the statistical studies reported in this paper we conclude that: 
1. Touchdown path lengths of $L \geq 10 \mathrm{~km}$ (all $\mathrm{F} j, j \geq 0$ ) are a good measure for the intensity of severe tornadoes.

2. The total continental USA path length of severe tornadoes $(L \geq 10 \mathrm{~km})$ during a convective day (12:00-12:00 UTC) is a good measure of the intensity of a severe tornado outbreak.

3. There are strongly non-Gaussian frequency-length statistics for:

- Touchdown path lengths of severe tornadoes $(L \geq 10 \mathrm{~km})$.

- Convective day total path lengths of severe tornado outbreaks.

- Path lengths for severe tornadoes during a single severe tornado outbreak.

4. Tornado path length statistics can be used to estimate the tornado hazard. This is in direct analogy (Schlelicke and Névir, 2011) to the way that the frequency-size statistics for earthquakes are used to quantify the earthquake hazard.

In conclusion, we believe that our studies provide evidence that tornado touchdown path lengths can be used as quantitative measures of the systematic properties of severe tornadoes and severe tornado outbreaks.

Acknowledgements. We thank reviewer Harold Brooks (NOAA) and an anonymous reviewer for their helpful comments which have improved this manuscript.

Edited by: J. Quaas

\section{References}

Brooks, H. E.: On the relationship of tornado path length and width to intensity, Weather Forecast., 19, 310-319, 2004.

Brooks, H. E. and Doswell III, C. A.: Some aspects of the international climatology of tornadoes by damage classification, Atmos. Res., 56, 191-201, 2001.

Doswell, C. A., Edwards, R., Thompson, R. L., Hart, J. A., and Crosbie, K. C.: A simple and flexible method for ranking severe weather events, Weather Forecast., 21, 939-951, 2006.
Doswell, C. A., Brooks, H. E., and Dotzek, N.: On the implementation of the Enhanced Fujita scale in the USA, Atmos. Res., 93, 554-563, 2009.

Fujita, T. T.: Proposed characterization of tornadoes and hurricanes by area and intensity. SMRP Research Paper 91, Dept. Geophys. Sciences, Univ. of Chicago, 42 pp., 1971.

Fujita, T. T.: Tornadoes and downbursts in the context of generalized planetary scales, J. Atmos. Sci., 38, 1511-1534, 1981.

Fujita, T. T. and Pearson, A. D.: Results of FPP classification of 1971 and 1972 tornadoes, Preprints, Eighth Conf. on Severe Local Storms, Denver, CO, Amer. Meteor. Soc., 142-145, 1973.

Glickman, T. S. (ed.): Glossary of Meteorology, 2nd edition, Amer. Meteor. Soc., 782 pp., 2000.

Hanks, T. C. and Kanamori, H.: A moment magnitude scale, J. Geophys. Res., 84, 2348-2350, 1979.

Humphries, N. E., Queiroz, N., Dyer, J. R., Pade, N. G., Musyl, M. K., Schaefer, K. M., Fuller, D. W., Brunnschweiler, J. M., Doyle, T. K., Houghton, J. D., Hays, G. C., Jones, C. S., Noble, L. R., Wearmouth, V. J., Southall, E. J. and Sims, D. W.: Environmental context explains Levy and Brownian movement patterns of marine predators. Nature, 465, 1066-1069, 2010.

Kelly, D. L., Schaefer, J. T., McNulty, R. P., Doswell, C. A., and Abbey Jr., R. F.: An augmented tornado climatology, Mon. Weather Rev., 106, 1172-1183, 1978.

McCarthy, D. W.: NWS tornado surveys and the impact on the national tornado database, First Symposium on F-Scale and Severe Weather Damage Assessment, Long Beach, CA, Amer. Meteor. Soc., preprint 3.2, 2003.

NOAA (National Oceanic and Atmospheric Administration): Storm Prediction Centre (SPC), Tornado, Hail, and Wind Database, available at: www.spc.noaa.gov/wcm/, last access: 30 July 2012.

Potter, S.: Fine-tuning Fujita, Weatherwise, 60, 64-71, 2007.

Richter, C.: An instrumental earthquake magnitude scale, Bull. Seis. Soc. Amer., 25, 1-32, 1935.

Schaefer, J. T., Schneider, R. S., and Kay, M. P.: The robustness of tornado hazard estimates, Third Symposium on Environmental Applications, Orlando, FL, USA, Amer. Meteor. Soc., paper 4.1, 35-41, 2002.

Schielicke, L. and Névir, P.: Introduction of an atmospheric moment combining Eulerian and Lagrangian aspects of vortices: Application to tornadoes, Atmos. Res., 100, 357-365, doi:10.1016/j.atmosres.2010.08.027, 2011.

Verbout, S. M., Brooks, H. E., Leslie, L. M., and Schultz, D. M.: Evolution of the U.S. tornado database: 1954-2003, Weather Forecast., 21, 86-93, 2006.

White, E. P., Enquist, B. J., and Green, J. L.: On estimating the exponent of power-law frequency distributions. Ecology, 89, 905-912 doi:10.1890/07-1288.1, 2008. 